\title{
Prevention of atherosclerosis by bioactive palmitoleate through suppression of organelle stress and inflammasome activation
}

\author{
Ismail Çimen, ${ }^{1,2}$ Begüm Kocatürk, ${ }^{1,2}$ Seda Koyuncu, ${ }^{1 *}$ Özlem Tufanlı, ${ }^{1,2}$ Umut I. Onat,,${ }^{1,2}$ \\ Asli D. Yıldırım, ${ }^{1,2}$ Onur Apaydın, ${ }^{1,2}$ Şeyma Demirsoy, ${ }^{1 \dagger}$ Zaliha G. Aykut, ${ }^{1}$ Uyen T. Nguyen, ${ }^{3}$ \\ Steven M. Watkins, ${ }^{3}$ Gökhan S. Hotamışlıgil, ${ }^{4}$ Ebru Erbay ${ }^{1,2 \ddagger}$
}

De novo lipogenesis (DNL), the conversion of glucose and other substrates to lipids, is often associated with ectopic lipid accumulation, metabolic stress, and insulin resistance, especially in the liver. However, organ-specific DNL can also generate distinct lipids with beneficial metabolic bioactivity, prompting a great interest in their use for the treatment of metabolic diseases. Palmitoleate (PAO), one such bioactive lipid, regulates lipid metabolism in liver and improves glucose utilization in skeletal muscle when it is generated de novo from the obese adipose tissue. We show that PAO treatment evokes an overall lipidomic remodeling of the endoplasmic reticulum (ER) membranes in macrophages and mouse tissues, which is associated with resistance of the ER to hyperlipidemic stress. By preventing ER stress, PAO blocks lipid-induced inflammasome activation in mouse and human macrophages.

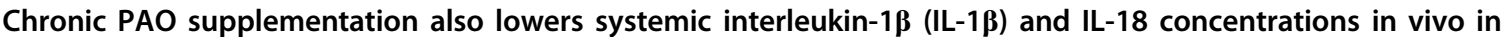
hyperlipidemic mice. Moreover, PAO prevents macrophage ER stress and IL-1 $\beta$ production in atherosclerotic plaques in vivo, resulting in a marked reduction in plaque macrophages and protection against atherosclerosis in mice. These findings demonstrate that oral supplementation with a product of DNL such as PAO can promote membrane remodeling associated with metabolic resilience of intracellular organelles to lipid stress and limit the progression of atherosclerosis. These findings support therapeutic PAO supplementation as a potential preventive approach against complex metabolic and inflammatory diseases such as atherosclerosis, which warrants further studies in humans.

\section{INTRODUCTION}

Fatty acids (FAs) can be generated de novo from a surplus of carbohydrates and other substrates in lipogenic organs. These lipids are then released as very low density lipoprotein (VLDL) particles from the liver and stored as triglycerides (TAGs) in the adipose tissue until further need (1). Although diet is usually a sufficient source of FA for mammals, de novo lipogenesis (DNL) is essential during development, as exemplified by the embryonic lethality of genetic mouse models lacking key DNL enzymes, such as FA synthase and acetyl-coenzyme A carboxylase 1. Furthermore, a maternal fat-rich diet does not rescue the lethality, suggesting that certain structural or signaling lipid molecules essential for embryonic development can only be generated de novo (2-4). Recent studies have shown that several bioactive lipid species are produced by DNL, including endogenous ligands for nuclear receptors [1-palmitoyl2-oleoyl-sn-glycerol-3-phosphocoline and 1-stearoyl-2-oleoyl-snglycerol-3-phosphocoline, peroxisome proliferator-activated receptor $\alpha$ (PPAR $\alpha$ ) and alkyl ether lipids, and PPAR $\gamma]$, fatty acid-hydroxy fatty acids (FAHFAs), and palmitoleate (PAO), which have specific biological activities and exert potent metabolic effects (5-9).

'Department of Molecular Biology and Genetics, Bilkent University, Ankara 06800 Turkey. ${ }^{2}$ National Nanotechnology Research Center, Bilkent University, Ankara 06800, Turkey. ${ }^{3}$ Metabolon, Sacramento, CA 95691, USA. ${ }^{4}$ Department of Genetics and Complex Diseases and Sabri Ülker Center, Harvard T.H. Chan School of Public Health, Boston, MA 02115, USA.

*Present address: Department of Cellular and Molecular Medicine, Katholieke Universiteit Leuven, Leuven 3000, Belgium.

tPresent address: CECAD Cluster of Excellence: Cellular Stress Responses in AgingAssociated Diseases, University of Cologne, Cologne 50931, Germany.

‡Corresponding author. Email: eerbay@bilkent.edu.tr
One striking observation regarding the biological impact of DNLgenerated lipids was made in snakes. PAO concentrations spike in the plasma of Burmese pythons that are going through an extreme metabolic adaptation to a large meal after long periods of starvation. Palmitoleic acid ethanolamide (palmitoleoyl ethanolamide), a potent regulator of energy balance, is markedly increased in the fed pythons' gastrointestinal tract. During this adaptation, the pythons undergo remarkable organ growth, including a particularly striking increase in cardiac size. On the other hand, administration of a lipid mix that includes $\mathrm{PAO}$ can promote healthy heart growth in a physiological setting (10). Together, these findings suggest a surprising beneficial metabolic impact of a monounsaturated fatty acid (MUFA), PAO, previously not associated with any distinct biological function.

Adipose tissue DNL and PAO production is also activated in fatty acid-binding protein (FABP)-deficient mice, which are highly resistant to developing insulin resistance and atherosclerosis despite developing obesity and dyslipidemia on a high-fat diet $(7,11-15)$. Liver is the primary site for FA synthesis. Adipose tissue DNL is usually inactive, and the lipid composition at this site reliably reflects dietary lipid input (16). However, adipose tissue from FABP-deficient mice becomes refractory to dietary input and activates DNL to generate its own FA from glucose $(7,17,18)$. PAO generated from the adipose tissue can have regulatory effects on lipid metabolism in liver and improve glucose utilization in skeletal muscle $(7,17,18)$. Hence, $\mathrm{PAO}$ is considered to be a lipokine, a lipid signal serving at the adipose tissue-liver and adipose tissue-muscle axes. Additionally, exogenous $\mathrm{PAO}$ administration has favorable metabolic and anti-inflammatory effects in mice, sheep, and rats (19-22). 
Interpretation of association studies to translate these preclinical findings to humans has been challenging because PAO is generated in the setting of obesity and alongside hepatic DNL. Because hepatic DNL associates with ectopic lipid accumulation and insulin resistance, the rise in PAO in serum has been associated with metabolic disorder (23-25). The coupling of liver DNL to PAO production in obesity may be an adaptation to help offset the hazardous effects of excess intake of saturated lipids (such as on membranes and membrane-initiated signaling) $(11,26,27)$. Another complication in the analysis of these epidemiological studies is that PAO concentrations were measured from different lipid classes. For example, PAO in the nonesterified fatty acid (NEFA) fraction shows a positive correlation with insulin sensitivity, whereas esterified PAO in cholesterol ester (CE), diacylglycerol (DAG), and TAG correlates with fatty liver disease, insulin resistance, diabetes, and acute coronary disease $(23,24,28-30)$. In humans, circulating esterified PAO concentration mainly reflects hepatic DNL $(31,32)$. These seemingly discrepant results imply that the free form of PAO could have distinct bioactivity in humans, similar to mice (7). Mouse studies using an exogenous source of PAO (independent of hepatic DNL) provide valuable insight into PAO's biological impact, including enhanced insulin sensitivity, reduced inflammation, and improved lipid metabolism $(7,21,22,33-35)$. In a recent randomized, controlled trial in humans, purified PAO treatment markedly reduced high-sensitivity C-reactive protein and improved serum lipids, suggesting that PAO supplementation may have a beneficial impact on atherosclerosis (36). However, these correlations do not establish causality or accurately reflect the real biological function of PAO in cardiovascular disease, warranting a direct analysis of the effect of PAO supplementation in a model of atherosclerosis.

Atherosclerosis is a chronic inflammatory disease of the arterial wall induced by lipid imbalance and a maladaptive immune system (37-39). The immune system plays a crucial role in the development of plaques in the intima, progression, and predisposition to rupture (40-42). The activation of the Nod-like receptor (NLR) family, pyrin domain containing-3 (NLRP3) inflammasome, a multiprotein complex in the myeloid linage that plays an important role in the innate immune response to pathogens, can be induced by cholesterol crystals or saturated FA (SFA) and promote atherogenesis $(43,44)$. The inflammasome is assembled from NACHT, LRR, and PYD domains-containing protein (NALP), apoptosis-associated speck-like protein containing a caspase1 recruitment domain (ASC), and caspase- 1 . Upon its activation, caspase1 processes pro-interleukin-1 $\beta$ (pro-IL-1 $\beta$ ) and pro-IL-18 into their mature forms, resulting in their secretion (45). Inflammasomes can also be activated in response to organelle stress, a pathological mechanism that contributes to atherogenesis $(11,44,46-48)$. Endoplasmic reticulum (ER) stress is linked to inflammasome activation through mobilization of intracellular calcium and generation of reactive oxygen species (ROS), which provide an activation signal for the inflammasome $(44,46,47)$. Moreover, calcium mobilization during ER stress causes mitochondria damage and generation of ROS linked to inflammasome activation (47). Under conditions of metabolic challenge, simultaneous ER stress and mitochondrial oxidative stress can generate a vicious cycle, potentiating inflammasome activity and establishing a pattern of chronic inflammation. Macrophage ER stress, similar to inflammasome activity, contributes to the development of obesity, insulin resistance, and atherosclerosis $(11,43)$. Additionally, macrophage mitochondrial oxidative stress promotes atherogenesis (48). In an earlier study, we have demonstrated that lipid-induced ER stress can be antagonized by PAO in vitro (11).
Whether PAO can inhibit inflammasome activation in macrophages or alter the course of atherosclerosis remains unknown.

Despite major interest in DNL-based therapeutic development for chronic metabolic diseases, nothing is known about the DNLgenerated lipid species' impact on atherosclerosis. Moreover, very little is known about the mechanism of action of these lipids in vivo, particularly in innate immune cells like macrophages, during the development of atherosclerosis. Here, we investigated the direct impact of a DNL product, PAO, on atherosclerosis. PAO prevented lipid-induced stress response in the ER, suppressing inflammasome activation in mouse and human macrophages and IL-1 $\beta$ and IL-18 production in vivo. The metabolic resilience of organelles to lipotoxic stress was associated with PAO-initiated lipidomic remodeling of ER membranes in macrophages and tissues in vivo. Overall, PAO supplementation increased the incorporation of this FA into major lipid species represented in the mouse aortas and reduced macrophage ER stress and atherosclerotic plaque area in apolipoprotein E-deficient $\left(A p o E^{-/}\right)$mice. These findings have potentially important translational implications for atherosclerosis treatment through nutritional management of bioactive lipid species such as PAO.

\section{RESULTS}

\section{PAO blocks lipid-induced inflammasome activation and inflammation in macrophages}

Here, we assessed the impact of PAO on SFA-induced inflammasome activation in macrophages. Earlier studies showed that treatment of lipopolysaccharide (LPS)-primed mouse bone marrow-derived macrophages (BMDMs) with an SFA such as palmitate (PA) specifically induced the activation of NLRP3 inflammasome and the secretion of IL-1 $\beta$ (44). PAO cotreatment completely blocked PA-induced IL- $1 \beta$ secretion $(P<0.001$; Fig. 1A). PA stimulation of LPS-primed BMDM activated caspase-1, whereas cotreatment with PAO blocked this activity (Fig. 1B). Priming with LPS up-regulates pro-Il-1 $\beta \mathrm{mRNA}$, which is expressed in low amounts in resting macrophages (49). PAO partially blocked the LPS-induced $I l-1 \beta$ mRNA increase in mouse BMDM but not in human THP-1 macrophages (Fig. 1, C and D). PA treatment enhanced $I l-1 \beta$ mRNA expression in LPS-primed BMDM and in THP-1 macrophages, but this effect could be completely blocked with PAO cotreatment (Fig. 1, C and D). Upon release, IL-1 $\beta$ and IL-18 engage their respective receptors on other innate immune cells and stimulate a proinflammatory response characterized by the secondary release of cytokines such as tumor necrosis factor- $\alpha$ (TNF $\alpha$ ) (50). PAO treatment blocked PA-stimulated Tnfa production in LPS-primed BMDM and human THP-1 macrophages $(P<0.001$; Fig. $1, \mathrm{E}$ and F). We observed similar inhibitory effects of PAO on PA-stimulated $\mathrm{Il}-6$ and $\mathrm{Ccl} 2$ production from LPS-primed BMDM and THP-1 cells (fig. S1, A to D). Finally, PAO inhibited PA-induced IL- $1 \beta$ secretion in LPS-primed human peripheral blood mononuclear cells (PBMCs) without reduction in $I L-1 \beta$ mRNA $(P<0.001$; Fig. $1 \mathrm{G}$ and fig. S1, E and F). Furthermore, PAO blocked PA-induced TNF $\alpha$ mRNA induction in the same cells $(P<$ 0.001; Fig. 1H) (51). Collectively, these experiments demonstrate that $\mathrm{PAO}$ is a potent inhibitor of lipid-induced inflammasome activation and proinflammatory cytokine production in mouse and human macrophages.

We next asked whether PAO could inhibit other inflammasome complexes. For this, mouse BMDMs were treated with specific activators 
Fig. 1. PAO prevents lipid-induced inflammasome activation and proinflammatory cytokine expression in macrophages. (A) Concentrations of secreted IL-1 $\beta$ in supernatants of LPS-primed BMDM stimulated with PA or $\mathrm{PAO}$, alone or together $(n=8)$. (B) Immunoblots for active caspase-1 (p10) from supernatant and pro-caspase1 (p45) and actin from cell lysate of BMDM. Blots shown are representative of three independent experiments. (C and D) Quantitative reverse transcription polymerase chain reaction (qRT-PCR) for (C) II-1 $\beta$ mRNA in mouse BMDM and (D) IL-1 $\beta$ in human THP-1 macrophages, primed with LPS and stimulated with PA or PAO, alone or together $(n \geq 3)$. (E and F) qRT-PCR for (E) Tnfo in mouse BMDM and (F) TNF $\alpha$ in human THP-1 macrophages, primed with LPS and stimulated with PA or PAO, alone or together $(n \geq 3)$. ( $\mathbf{G}$ and $\mathbf{H}$ ) LPS-primed human PBMCs were analyzed $(\mathrm{G})$ by enzyme-linked immunosorbent assay (ELISA) for IL-1 $\beta$ secreted into conditioned medium $(n \geq 3)$ and $(\mathrm{H})$ by qRTPCR for TNF $\alpha$ mRNA $(n \geq 3)$. (I and J) Active caspase-1 (p10) and mature IL-1 $\beta$ were detected by Western blotting in supernatant of mouse BMDMs primed with LPS and induced with various (I) NLRP3 agonists (ATP, chitosan, or alum) or (J) specific activators of other inflammasome complexes [poly(dA:dT), flagellin, and MDP] according to previously published protocols (91-95). Blots shown are representative of three independent experiments. Data are means \pm SEM. ${ }^{*} P<0.05,{ }^{* *} P<0.01,{ }^{* * *} P<$ 0.001 . One-way analysis of variance (ANOVA) [in (C) to $(\mathrm{H})$ ] or two-tailed Mann-Whitney $U$ test [in (A)] was used for statistical analysis.

for various inflammasome complexes (52). Although PAO abolished lipid-induced NLRP3 inflammasome activation, as evidenced by the suppression of cleaved caspase- 1 and secreted IL-1 $\beta$, it did not inhibit NLRP3 inflammasome induction by aluminum crystals (alum), chitosan, or adenosine triphosphate (ATP) (Fig. 1I). These findings showed that PAO specifically intercepts lipidinduced NLRP3 inflammasome activation. Moreover, PAO did not prevent activation of the absent in melanoma 2 (AIM2) inflammasome by poly(deoxyadenylicdeoxythymidylic) [poly(dA:dT)] acid, NLR family caspase-1 recruitment domain (CARD)-containing protein-4 (NLRC4) inflammasome by flagellin, or NLRP1 inflammasome by muramyldipeptide (MDP), supporting PAO's specific role as an inhibitor of the lipid-induced NLRP3 inflammasome (Fig. 1J).

\section{PAO prevents PA-induced ER stress by remodeling ER membranes}

Next, we investigated the mechanism by which PAO blocks lipid-induced NLRP3 inflammasome activation. SFAs like PA can induce ER stress and activate the inflammasome $(11,44,53)$. Previous studies showed that ER stress induces inflammasome activation through several mechanisms, including calcium mobilization, ROS release, and promotion of mitochondrial damage (52). Damaged mitochondria release ROS, which can further potentiate the inflammasome activity $(44,47)$. We hypothesized that inhibition of PA-induced
A

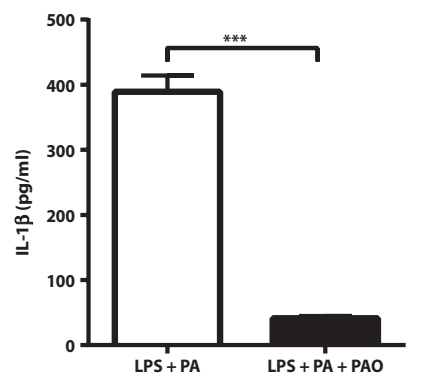

C

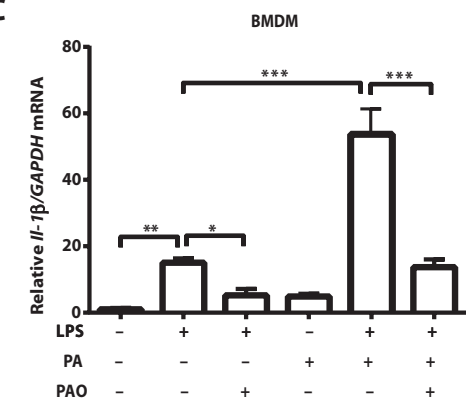

E

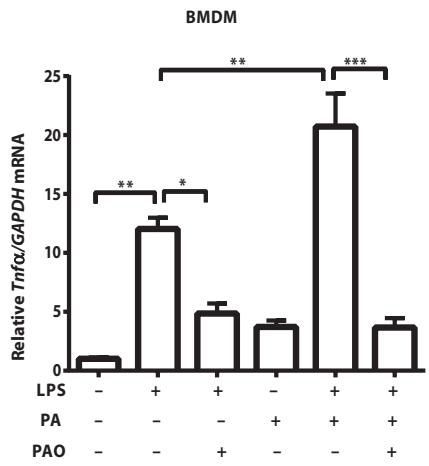

G

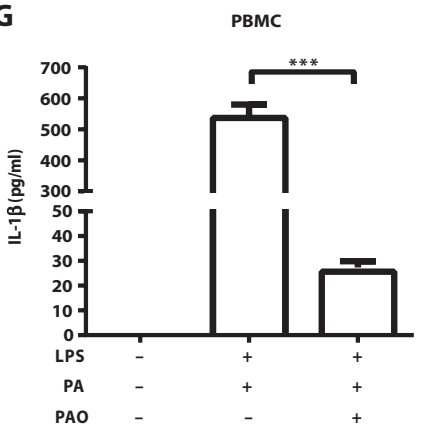

\section{I}

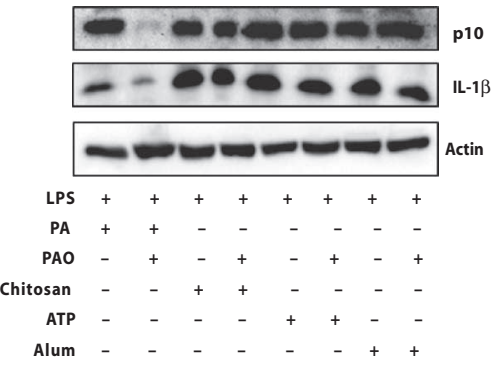

B

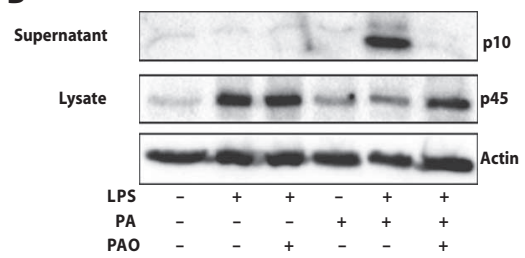

D

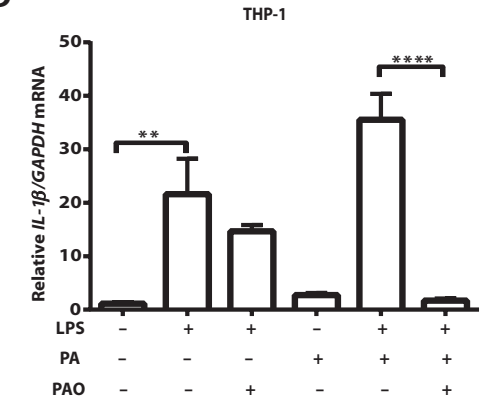

$\mathbf{F}$

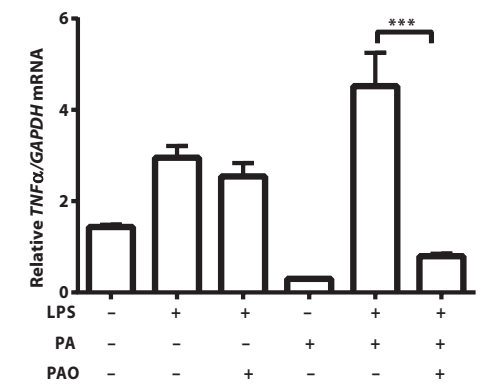

H

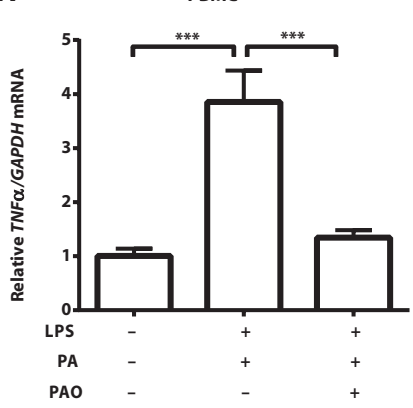

J

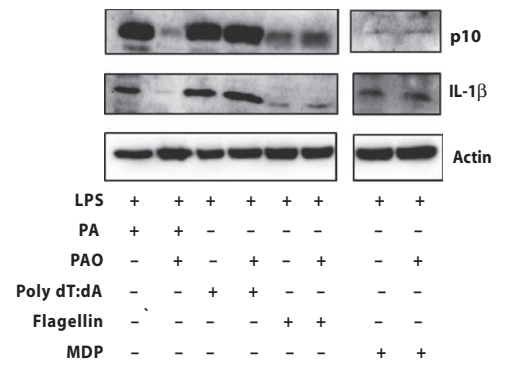


Fig. 2. PAO regulates lipid composition in primary mouse macrophages. Mouse BMDMs were treated with $\mathrm{PA}$ or $\mathrm{PAO}$, alone or together, for 6 hours. (A) Mean PAO (C16:1n7) concentration [mole percent ( $\mathrm{mol} \%)$; the ratio of moles of FAs to total moles of FAs]. (B) MUFA/SFA ratio. (C) PAO (C16:1n7) concentration (mol \%) in various lipid classes. (D) Mean concentration of each lipid class $(\mu M)$. ns, not significant. (E) Mean concentration of PA (C16:0) in each lipid class $(\mu \mathrm{M})$. (F) PAO (C16:1n7) concentration in various $\mathrm{PLs}$ ( $\mathrm{mol} \%)$. Data are means \pm SEM. ${ }^{*} P<0.05,{ }^{* *} P<0.01,{ }^{* * *} P<0.001 . n=4$. Nonparametric Kruskal-Wallis test and unpaired two-tailed Student's $t$ test were used for statistical analysis. PC, phosphatidylcholine; LPC, lysophosphatidylcholine; LPE, lysophosphatidylethanolamine; PG, phosphatidylglycerol; $\mathrm{PE}$, phosphatidylethanolamine; $\mathrm{PI}$, phosphatidylinositol; PS, phosphatidylserine; FFA, free fatty acid.

ER stress by PAO blocks NLRP3 inflammasome activation in macrophages. In an earlier study, we showed that PAO prevents lipid-induced ER stress but not proteotoxic ER stress in macrophages (11). Because inflammasome activation requires LPS pretreatment in cultured macrophages, we assessed whether PAO can block ER stress under inflammasome-activating conditions. PAO also blocked PA-induced ER stress in LPS-pretreated BMDMs, as measured by spliced Xbp1 mRNA production (fig. S1G). This finding shows that PAO can block lipid-induced ER stress upstream of inflammasome activation.

To understand how PAO limits the impact of lipid stress on macrophages, we next used high-resolution quantitative lipidomics to analyze PAO-induced alterations in macrophage lipid composition. For this purpose, we analyzed the lipid changes in mouse BMDMs treated with PA and $\mathrm{PAO}$ alone or in combination (PA + PAO). Analysis of the data showed increases in total PAO concentration and MUFA/SFA ratio in cells treated with PAO (alone or with PA; $P<0.001$; Fig. 2, A and B). PAO incorporated into all major lipid species whether applied alone or in combination with PA (Fig. 2C). PA + PAO cotreatment expanded the TAG compartment and its metabolic precursor, DAG, suggesting that PAO may be buffering PA-associated lipotoxicity by facilitating its effective entry into neutral lipid stores $(P<0.001$ and $P<0.01$, respectively; Fig. 2D). More PA incorporated into TAG and DAG when cotreated with PAO $(P<$ 0.001 and $P<0.01 ;$ Fig. 2 E). We also examined another membrane constituent, phospholipids (PLs), because their production is mainly regulated by the ER with the contribution of IRE1-XBP1 signaling branch (54). PA, but not PAO (alone or cotreated), expanded the PL compartment $(P<0.01$; Fig. 2D). However, PAO effectively incorporated into all PL species, with highest concentrations seen in phosphatidylcholine

C

$\mathbf{F}$
B
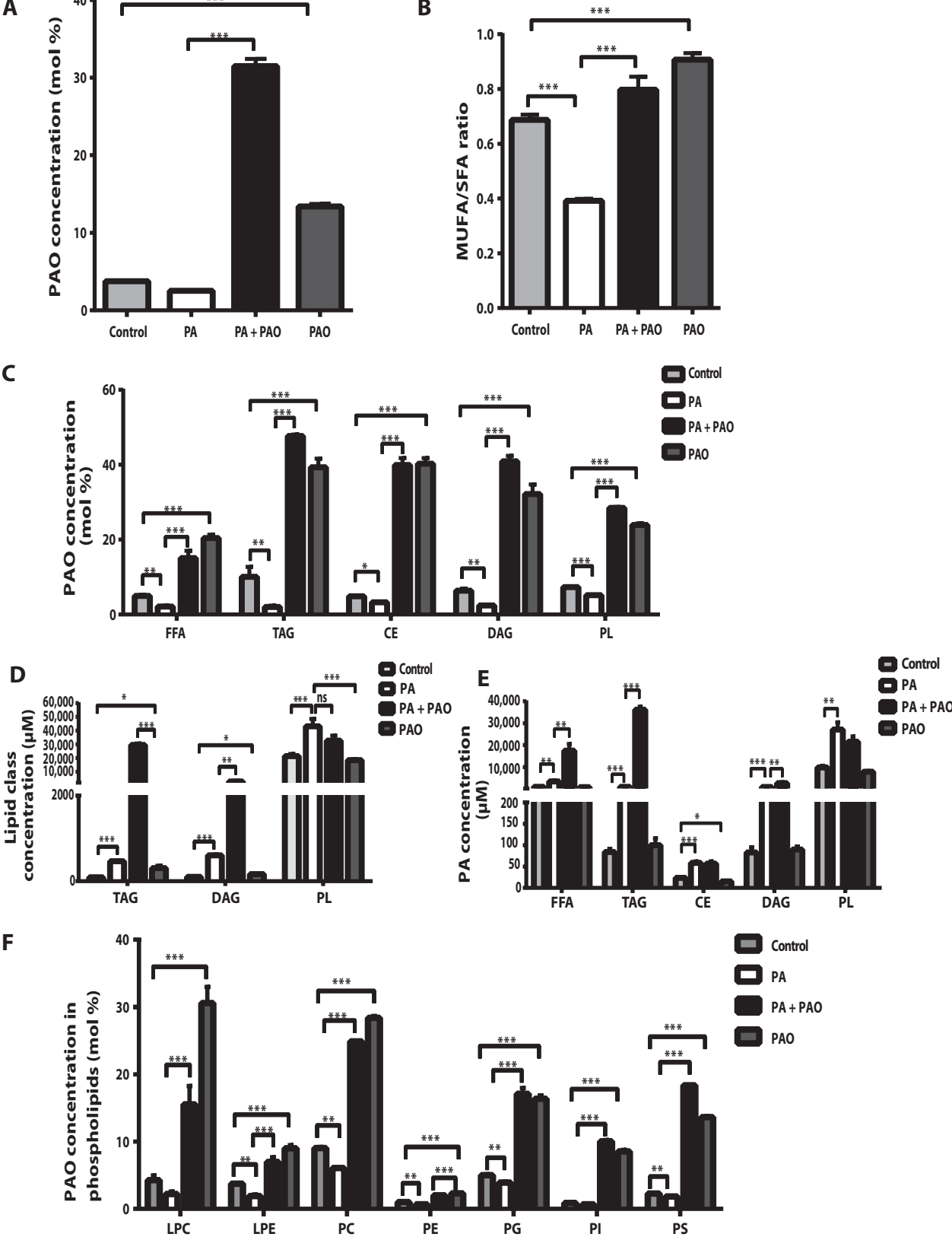

and its metabolic precursor, lysophosphatidylcholine (Fig. 2, C and F). These findings imply that desaturation of membrane lipids by PAO could have an influence on the membrane's biophysical properties (55).

Next, we used a mouse model of atherosclerosis, $\mathrm{ApoE}^{-/-}$mice, in which ER stress and inflammasome activation can be induced over time by a hyperlipidemic (Western) diet (43). Typically, PAO makes up about $0.38 \%$ of Western diet. We decided to administer up to threefold higher PAO by oral gavage (about $1.2 \%$ of diet or $1400 \mathrm{mg} / \mathrm{kg}$ per day) to $A p o E^{-/-}$mice on Western diet and analyze the effect of this chronic exposure on lipid composition of plasma and multiple tissues (56). Four weeks of PAO treatment increased $\mathrm{PAO}$ in the circulation and aortas of $A p o E^{-1-}$ mice but not in liver, adipose, and muscle tissues $(P<0.05$; Fig. $3 \mathrm{~A}$ and fig. S2A). In addition, the MUFA/SFA ratio was increased in the aortas and plasma from $\mathrm{PAO}$-treated mice when compared to control 
A

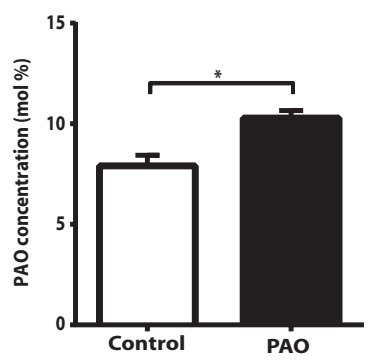

B

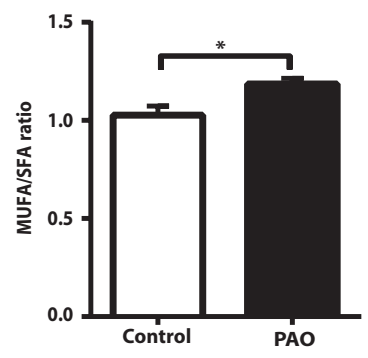

C

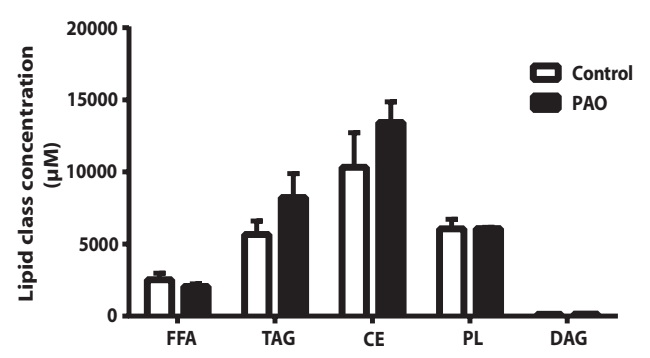

D

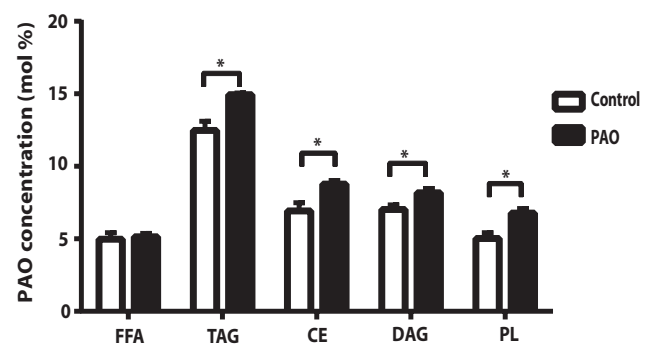

E

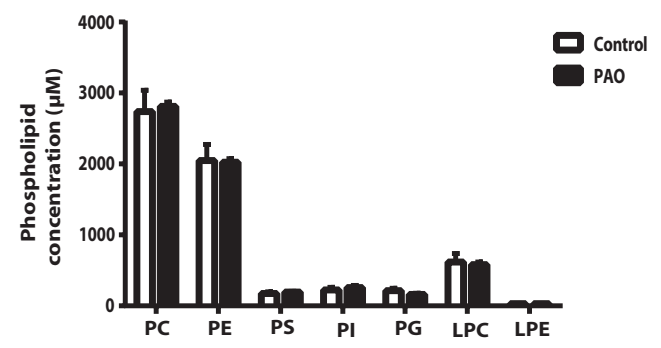

$\mathbf{F}$

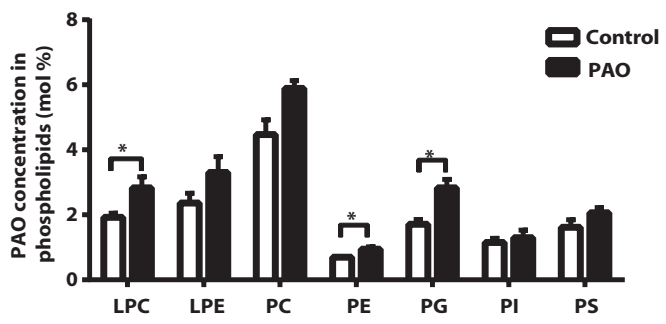

I

Aorta-CE
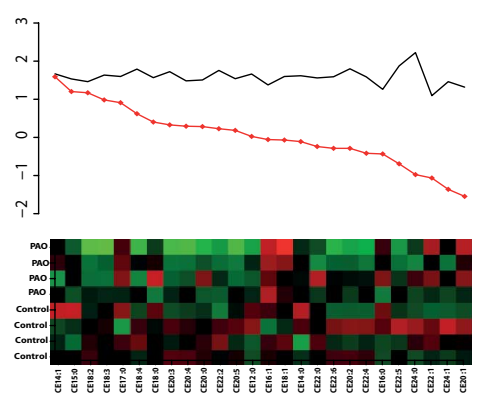

K

Aorta-DAG
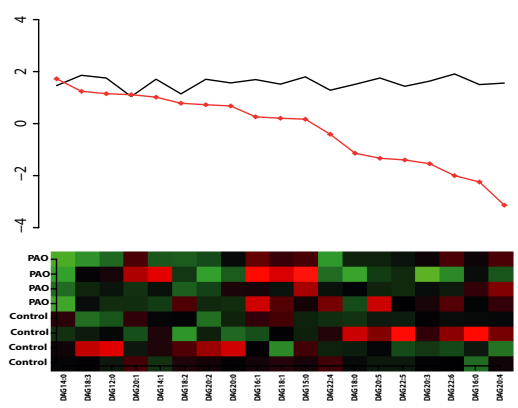

Fig. 3. PAO regulates lipid composition in aortas from $\mathrm{ApoE}^{-/-}$mice. Aortas were isolated from PAO-treated and control $A p o E^{1-}$ mice on Western diet and analyzed. (A) Mean concentration of PAO (C16:1 n7) (mol \%; the ratio of moles of FAs to total moles of FAs). (B) MUFA/SFA ratio. (C) Concentration of each lipid metabolite (expressed as $\mu \mathrm{M}$ ). (D) Mean concentration of PAO $(\mathrm{C} 16: 1 \mathrm{n7})(\mathrm{mol} \%)$ in various lipid metabolites. (E) Concentration $(\mu \mathrm{M})$ of the various PL metabolites. (F) Mean concentration of C16:1n7 (mol \%) in the various PL metabolites. (G to $\mathbf{K}$ ) The heat maps display lipid class composition analysis for (G) PG, (H) PE, (I) CE, (J) TAG, and (K) DAG. In the heat maps, $x$ axis shows various lipid metabolites in each sample ( $y$ axis) that are up-regulated (red) or down-regulated (green), calculated by centering to the mean of the control group and scaled by the SD of all observations. In the line plots, $y$ axis shows the $t$ statistics from comparing two groups. The black line is the 95th percentile of the standardized statistics over 1000 permutations. The red dots are the $z$ scores of the observed statistics that were standardized to 1000 permutations. Graphs show means \pm SEM. ${ }^{*} P<0.05 . n=4$. Unpaired two-tailed Student's $t$ test [in (B)] or Wilcoxon test [in (A) and (C) to (F)] was used for statistical analysis. 
$(P<0.05$, Fig. 3B; $P<0.01$, fig. S2B). PAO treatment did not cause expansion of any major lipid class analyzed in aortas and plasma (Fig. 3C and fig. S2C), but PAO was increased in these samples $(P<0.05$; Fig. 3D and fig. S2D). In particular, PAO was increased in many of the PL species without expanding the PL compartment (Fig. 3, E and F, and fig. S2, E and F). PAO (C16:1n7) was one of the most elevated FAs in many of the lipid compartments (Fig. 3, G to K, and fig. S3, A to F). In conclusion, oral PAO supplementation increased plasma PAO concentration and enhanced PAO incorporation into various lipid compartments in the aortas of hyperlipidemic mice.

PAO-induced lipidomic remodeling of cellular membranes and tissues may underlie the resilience of ER to lipotoxic stress. Lipotoxicity, lipid-induced cellular dysfunction and death, is associated with the disruption of ER structure resulting from membrane stiffening caused by increased SFA or cholesterol content $(57,58)$. Therefore, we examined whether orally delivered PAO can enter and remodel ER membranes in mouse tissues. Lipidomic analysis of isolated liver ERs from PAOtreated mice showed a significant increase in total PAO in ER membranes compared to control ( $P<0.05$; Fig. $4 \mathrm{~A}$ and fig. S4A). PAO treatment increased the MUFA/SFA ratio in liver ERs (Fig. 4B). PAO incorporated into many lipid compartments, especially PL and CE, of liver ERs $(P<$ 0.05 and $P<0.01$; Fig. 4C). PAO was one of the most highly elevated FAs in all lipid compartments of liver ER (Fig. 4, D and E, and fig. S4, B to D). Collectively, these data demonstrate that oral PAO treatment can promote systemic desaturation of ER membranes in vivo.

Lipidomic analysis of ER compartments from macrophages also reflects the increase in total $\mathrm{PAO}$ and MUFA/SFA ratios after PAO treatment $(P<0.05$, Fig. 4F; $P<0.01$, Fig. 4G; and validation of the ER fraction; fig. S4E). PAO is incorporated into all ER lipid species (Fig. 4H). In the macrophages, PA-induced unfolded protein response (UPR) activation (as assessed by IRE1 phosphorylation and spliced XBP1 protein) was blocked by PAO (fig. S4F). Therefore, the efficient integration of PAO into PL and CE together with an increase in MUFA/SFA ratio in the ER membranes suggests that PAO treatment could promote robust changes in membrane dynamics and membrane-initiated UPR activation in macrophages. For example, the assembly of IRE1 or PERK into high-order oligomers on ER membranes is essential for transmitting stress from within this organelle (59). Activation of these proximal UPR kinases by saturated lipids, unlike activation by unfolded proteins, does not require ligand binding by their luminal domains (60). Lipid composition changes in ER membrane may alter oligomerization and activation states of these kinases. Thus, we investigated the impact of PA and PAO on IRE1 oligomerization states on ER membranes using previously established protocols (61). PA induced green fluorescent protein (GFP)-tagged IRE1 oligomer formation on ER and splicing of XBP1, which was largely prevented by cotreatment with PAO (Fig. $4 \mathrm{I}$ and fig. S4, G to I). This result confirms that ER membrane saturation or desaturation by PA or PAO, respectively, can alter the oligomerization state of a proximal UPR sensor and affect downstream UPR signaling.

\section{PAO prevents the development of atherosclerosis in mice}

Atherosclerosis is a nonresolving chronic inflammatory disease of the arterial wall. It is triggered by subendothelial deposition of lipoproteins in areas of disturbed laminar flow that cause endothelial damage. These retained lipoproteins are susceptible to oxidation and other modifications that render them highly proinflammatory and toxic to the overlying endothelial cells. This also causes activation of the endothelial cells and intramural recruitment of cells from the mononuclear lineage. In the subendothelial space, monocytes are transformed first to phagocytes that scavenge lipid deposits and then into proinflammatory, cholesterolladen foam cells. The foam cells eventually become engorged with cholesterol and lose their capacity to migrate, compromising their immune response. As a result, the plaques advance and become more complex with the contribution of other immune cells and vascular smooth muscle (VSM) cells. At this advanced stage, the plaque stability is highly influenced by macrophages through their secretion of proinflammatory cytokines and chemokines as well as matrix-degrading proteases. Moreover, upon their death, the release of their toxic content results in the formation of a prothrombotic necrotic core $(37-39,62)$.

Atherosclerotic plaques of both mice and humans display signs of ER stress, mitochondrial oxidative stress, and inflammasome activation in macrophages $(11,43,63-66)$. Increased ER stress in lesions associates with plaque vulnerability and acute coronary syndrome (65). Genetic, dietary, and chemical interventions that reduce ER stress and inflammasome activity can prevent atherosclerosis in mice $(11,67-70)$. Moreover, specific and selective inhibitors for ER stress and inflammasomes that can alleviate multiple components of metabolic syndrome are currently under investigation $(52,71)$.

Given our findings that PAO can inhibit lipid-induced stress in the ER and subsequent activation of the inflammasome in macrophages (Fig. 1 and fig. S1), we hypothesized that chronic PAO intervention in mice could prevent atherosclerosis in vivo. For this purpose, male $A p o E^{-/-}$mice were placed on a hypercholesterolemic diet at 6 weeks of age for a total of 16 weeks. There were no significant differences in body weights or blood glucose concentrations of mice in the experimental groups before or after PAO treatment (table S1). Starting at 12 weeks on diet, PAO was administered daily by oral gavage, whereas control mice received vehicle for a total of 4 weeks (Fig. 5A). Chronic oral PAO treatment resulted in a significant $(P<0.01)$ decrease $(-33 \%)$ in the development of atherosclerotic lesions in en face aorta analysis when compared to control mice (Fig. 5B). The impact of PAO on atherosclerosis development was also evaluated in cross-sectional lesions of the aortic root. The foam cell area in the plaques (visualized with Oil Red O staining) was significantly $(P<0.05)$ decreased $(-29.4 \%)$ in the aortic sinus sections (Fig. 5C). In parallel, there was a significant $(P<0.01)$ reduction in the necrotic core area in the lesions of the PAOtreated group when compared to control mice (-31\%) (Fig. 5D). These results show that PAO treatment alleviated atherosclerosis without improving dyslipidemia and that ER stress (as evident by decrease in $s X b p 1$ mRNA in hearts) was markedly reduced in PAO-treated mice (Fig. 5E and fig. S5, A to D). These in vivo findings demonstrate that dietary supplementation with PAO can effectively mitigate atherosclerosis in mice.

\section{PAO alters plaque composition by reducing macrophages in lesions}

Many kinds of immune and nonimmune cell types participate in the formation and advancement of atherosclerotic plaques. For example, intramural deposition of lipoproteins is linked to endothelial damage that triggers mononuclear cell infiltration to the subendothelial space and subsequent formation of foam cells. Other immune cell types, such as dendritic cells, lymphocytes, eosinophils, and mast cells, are also involved in plaque advancement $(38,39,68,72,73)$. Given that PAO reduces atherosclerosis in $A p o E^{-1-}$ mice, we next analyzed PAO-initiated changes in the cellular composition of atherosclerotic plaques. When compared to control, PAO treatment reduced $(-18 \%)$ the macrophage 
Fig. 4. PAO regulates lipid composition in the ERs from livers of $A p O E^{-/-}$mice and from macrophages. (A to E) Liver ER fractions were isolated from PAO-treated and control $\mathrm{ApoE}^{-1}$ mice on Western diet and analyzed. (A) Mean concentration of PAO (C16:1n7) (mol \%; the ratio of moles of FAs to total moles of FAs). (B) MUFA/SFA ratio. (C) Mean concentration $(\mu \mathrm{M})$ of PAO (C16:1n7) in various lipid metabolites. Data are means \pm SEM. ${ }^{*} P<$ $0.05,{ }^{* *} P<0.01 . n=5$. Unpaired two-tailed Student's $t$ test [in (B)] and Wilcoxon test [in (A) and (C)] were used for statistical analysis. (D and E) The heat maps display lipid class composition analysis for (D) PC and (E) CE. In the heat maps, $x$ axis shows each lipid metabolite measurement in each sample ( $y$ axis) as up-regulated (red) or down-regulated (green), as determined by centering to the mean of the control group and scaled by the SD of all observations. In the line plot, $y$ axis shows the $t$ statistics from comparing two groups. The black line is the 95th percentile of the standardized statistics over 1000 permutations. The red dots are the $z$ scores of the observed statistics that were standardized to 1000 permutations. Data are means \pm SEM. ${ }^{*} P<0.05,{ }^{* *} P<0.01 . n=5$. ( $\mathbf{F}$ to H) ER fractions were isolated from RAW 293.6 macrophages and analyzed. (F) Mean concentration (mol \%) of C16:1n7. (G) MUFA/SFA ratio. (H) Mean concentration (mol \%) of $\mathrm{C} 16: 1 \mathrm{n} 7$ in various lipid classes. Data are means \pm SEM. ${ }^{*} P<0.05,{ }^{* *} P<$ $0.01,{ }^{* * *} P<0.001 . n=4$. Nonparametric KruskalWallis test and unpaired two-tailed Student's $t$ test were used for statistical analysis. (I) Cells expressing GFP-IRE1 were treated with PA or PAO, alone or together, for 6 hours and analyzed for formation of discrete IRE1 oligomer foci (green dots) by imaging with a confocal microscope (blue, nuclei; green, GFP-IRE1). The graph depicts quantification of IRE1 oligomerization as reported distinct IRE1 foci (green) per cell. Data are means \pm SEM. ${ }^{* *} P<0.01 . n \geq 100$ cells from four experiments. To compare PA-treated versus cotreated with PA and PAO, Mann-Whitney $U$ test was used for statistical analysis. Scale bar, $20 \mu \mathrm{m}$. DAPI, 4',6-diamidino-2-phenylindole.

content of aortic root lesions, as visualized by MOMA-2 staining ( $P<0.05$; Fig. 6A and fig. S6). This is consistent with the reduced amount of foam cells in the lesions of PAO-treated mice (Fig. 5C, seen as Oil Red O-positive staining). This was not, however, related to an increase in macrophage apoptosis, because PAO treatment also reduced the number of apoptotic cells $(-34.8 \%)$ in the lesion area stained with MOMA2 , as determined by terminal deoxynucleotidyl transferase-mediated deoxyuridine triphosphate nick end labeling (TUNEL) assay $(P<0.05$; Fig. $6 \mathrm{~B})$. Furthermore, we examined the T lymphocyte populations in PAO-treated and control aortic sinus lesions and found no significant differences in $\mathrm{CD}^{+}$cells between the two experimental groups (Fig. 6C and fig. S6).
A

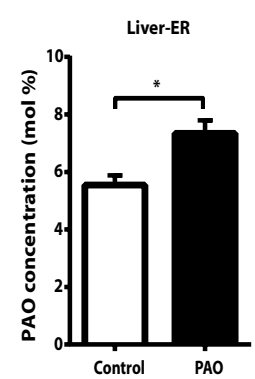

B
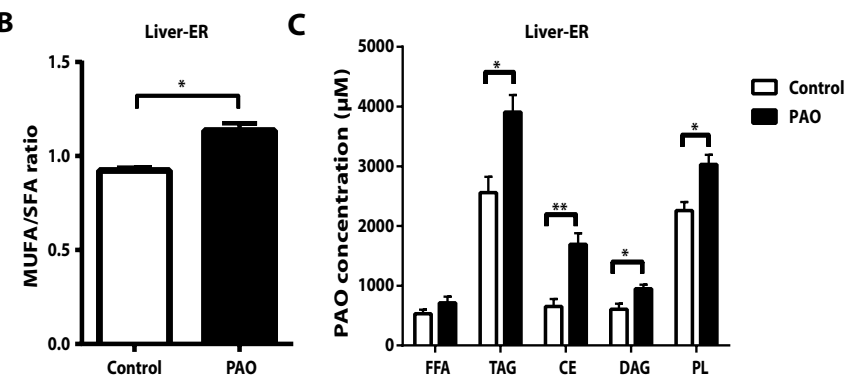

D

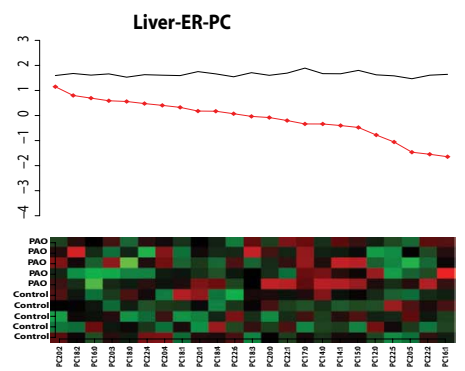

E
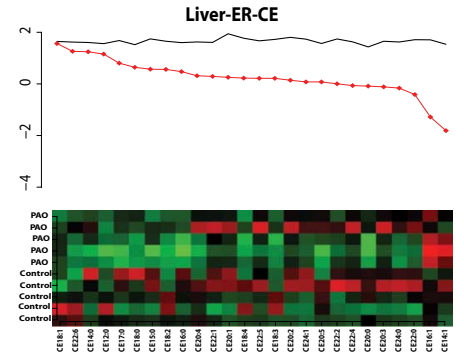

$\mathbf{F}$

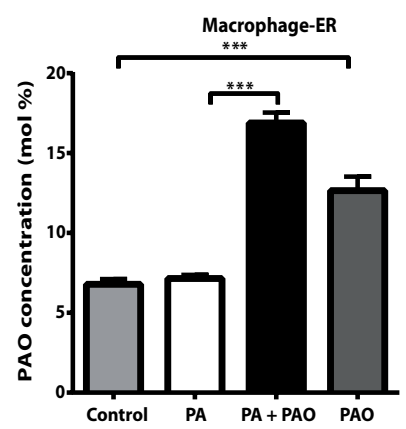

G

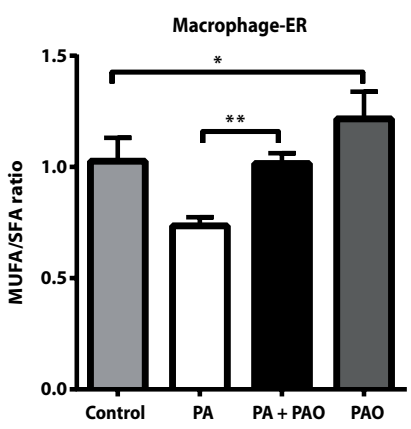

H
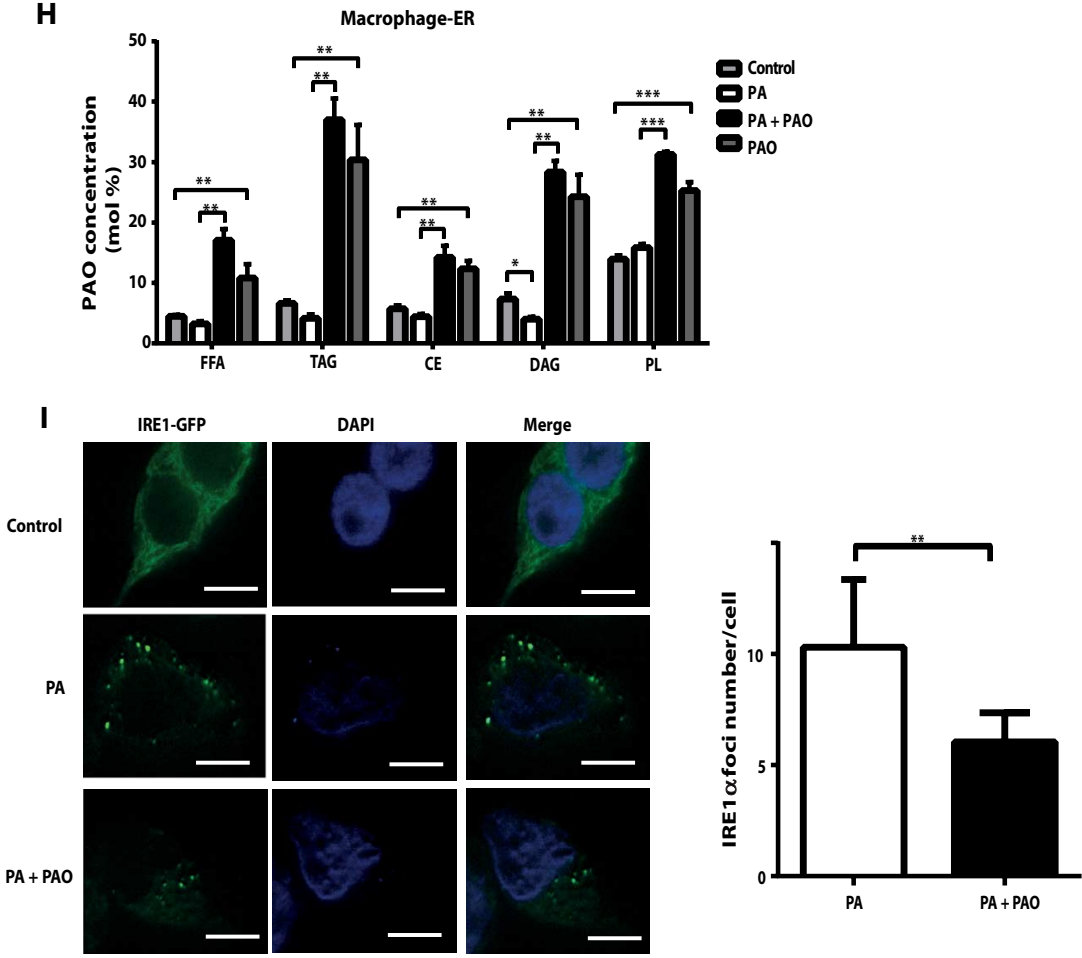
Fig. 5. PAO treatment reduces atherosclerosis in ApoE $^{-/-}$mice. (A) Experimental design for the analysis of atherosclerosis in $\mathrm{ApoE}^{-/-}$mice on a Western diet. BSA, bovine serum albumin; PBS, phosphate-buffered saline. (B) PAO treatment reduced atherosclerotic lesions in en face aorta (control, $n=18$; PAO, $n=15$; ${ }^{*} P<0.01$ ). (C) Histology of plaques (marked with arrows) at the aortic sinus stained with Oil Red $\mathrm{O}$ and hematoxylin and eosin (H\&E). Lesion area was reduced in the plaques of PAO-treated mice (control, $n=10$; PAO, $n=10$; ${ }^{*} P<$ $0.05)$. Scale bar, $300 \mu \mathrm{m}$. (D) The necrotic core area (marked with dashed lines and arrows) was also decreased in the plaques of PAO-treated mice as compared to control mice (control, $n=12 ; \mathrm{PAO} ; n=11$, ${ }^{* *} P<0.01$ ). Scale bar, $50 \mu \mathrm{m}$. (E) Serum total cholesterol and lipoprotein cholesterol [VLDL, low-density lipoprotein (LDL), or high-density lipoprotein (HDL)] concentrations were similar between PAO-treated and control mice. (F) Lipoprotein profiles from control (blue) and PAO-treated (red) $\mathrm{ApoE}^{1-}$ mice show the average distribution ( $\mu \mathrm{g}$ per fraction; 1 to $13: \mathrm{VLDL}, 14$ to $31: \mathrm{LDL}$, and 32 to $50: \mathrm{HDL}$ ) of total cholesterol for each group. Data are means \pm SEM. ${ }^{*} P<0.05,{ }^{* *} P<0.01$. ns, not significant. control, $n=4$; PAO, $n=6$. Control versus PAO: Mann-Whitney $U$ test [in (B) to (D)] or unpaired two-tailed Student's $t$ test [in (E)] was used for statistical analysis.

In addition to having proatherogenic immune cell types, atherosclerotic lesions contain VSM, which can migrate from adventitia to intima and aid in sealing the fibrous cap against rupture. This migratory effect is controlled by the mediators and chemokines released from macrophages and other plaque cells (38). Immunofluorescence staining of aortic root lesions with a specific antibody against $\alpha$-smooth muscle actin showed no significant change in $\mathrm{VSM}^{+}$area after PAO treatment (Fig. 6D and fig. S6). Additionally, VSM-derived collagen content showed no change in PAO-treated lesions when compared to control lesions (Fig. 6E). Overall, PAO treatment does not appear to improve the mechanical strength of the lesions.

\section{PAO prevents macrophage ER stress and inflammasome activation in lesions}

Our findings demonstrated a strong impact of PAO on lesion macrophages and their viability. Our earlier studies have also indicated that PAO can block lipotoxic, but not proteotoxic, ER stress in macrophages (11). In our current study, we observed that PAO can block lipid-induced ER stress, subsequently leading to suppression of the inflammasome (Fig. 1 and fig. S1). Hence, we asked whether the anti-stress and antiinflammatory effects of PAO observed in macrophages can be recapitulated in vivo during atherogenesis. First, we examined the immunofluorescence signal intensity for two markers of activation of the ER stress response, namely, phosphorylation of eukaryotic initiation factor $2 \alpha(\mathrm{eIF} 2 \alpha)$ and expression of cyclic adenosine monophosphate-dependent transcription factor 3 (ATF3) in macrophage-enriched areas of aortic root
A

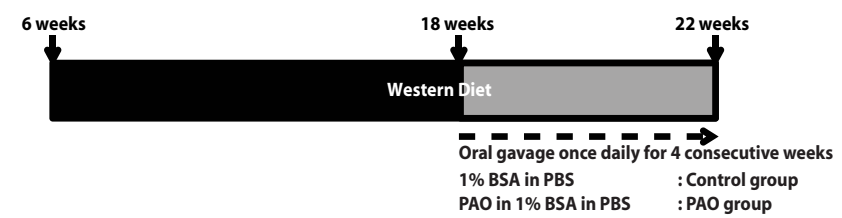

B
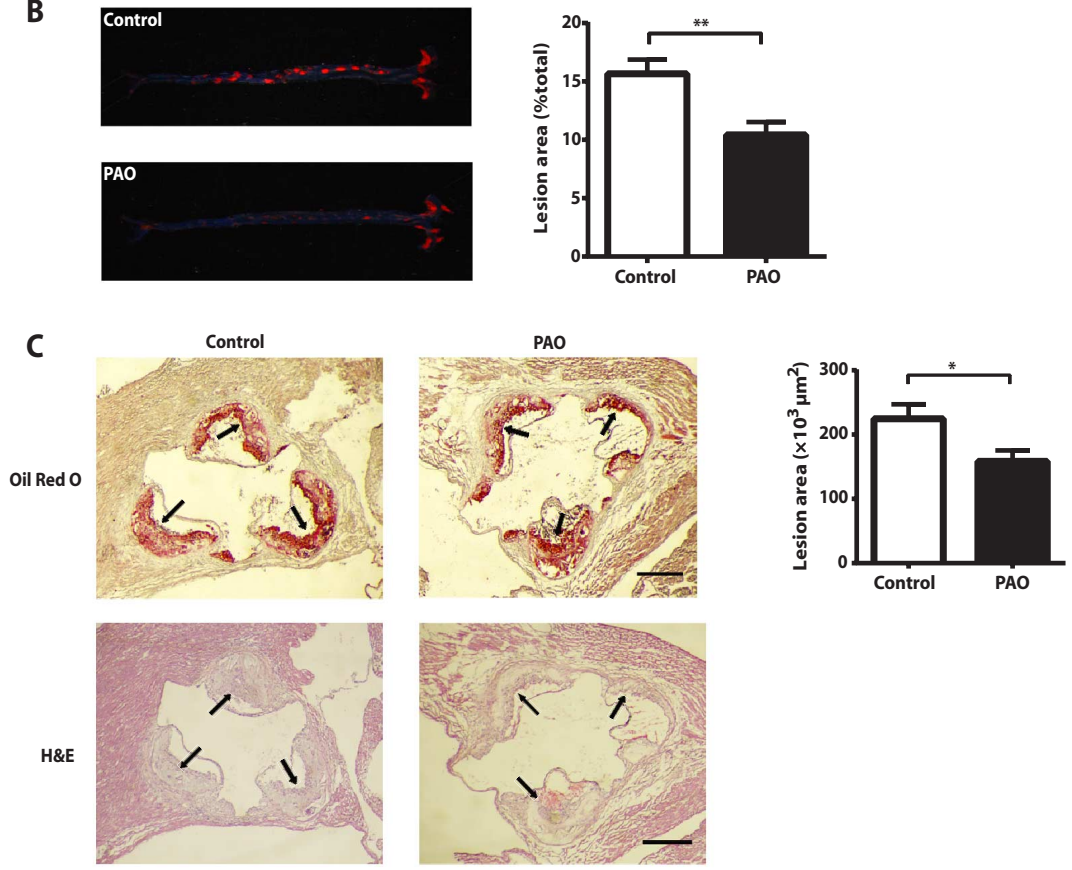

D
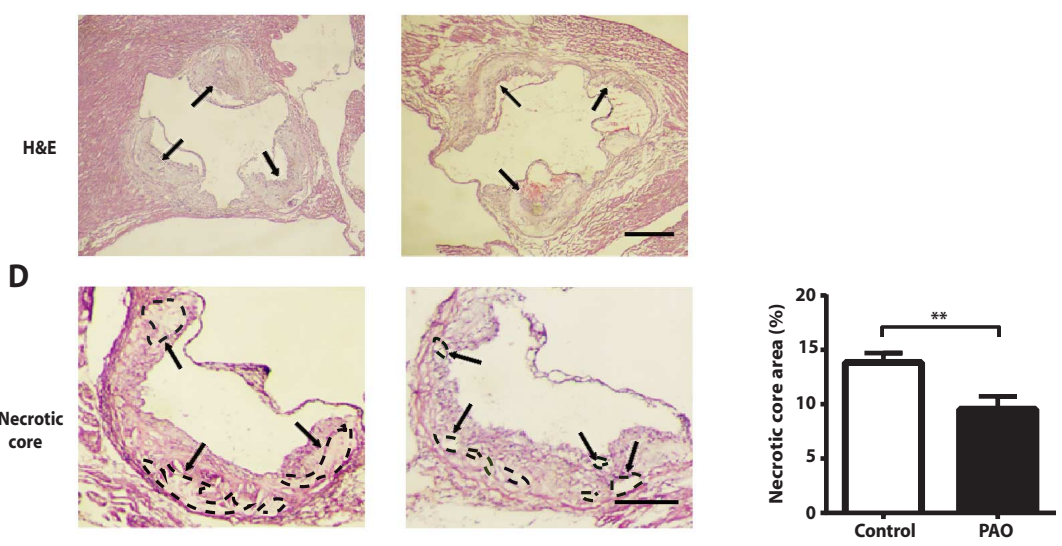

E
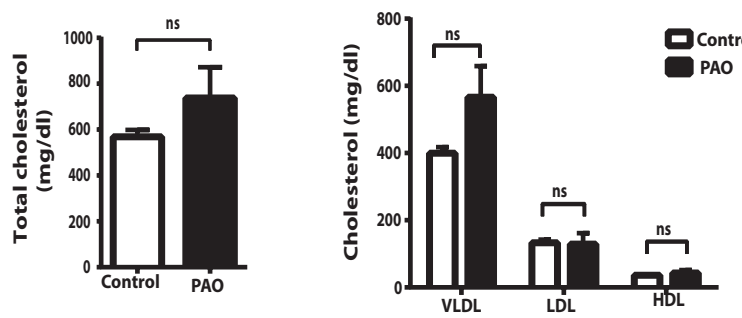

$\mathbf{F}$

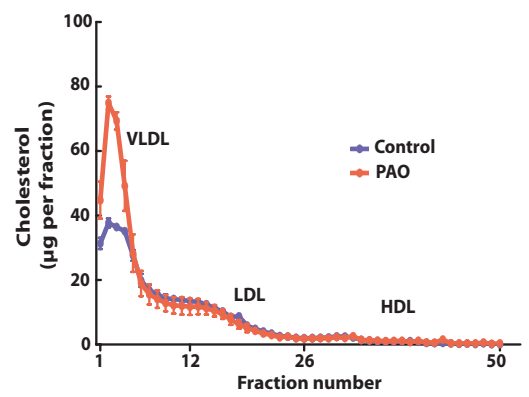


Fig. 6. PAO reduces the macrophage area and apoptotic cells in atherosclerotic plaques. Six-week-old $\mathrm{ApoE}^{-1-}$ mice were put on Western diet (total of 16 weeks) and orally gavaged with PAO or vehicle daily (for the last 4 weeks). Proximal aorta cryosections were stained with specific antibodies for various cellular markers. The representative images are shown for $(\mathbf{A})$ macrophage marker MOMA-2 ( $n=10$ per group). Mean percent MOMA-2-positive area relative to total lesion area is reported. (B) TUNEL assay. Arrowheads show apoptotic cells (control, $n=10 ; \mathrm{PAO}, n=$ 9). Average apoptotic cells per plaque area $\left(\mathrm{mm}^{2}\right)$ are reported. (C) T cell marker, CD3 (control, $n=9$; PAO, $n=10$ ). Arrowheads show CD3-positive cells. The average numbers of $\mathrm{T}$ cells per plaque area $\left(\mathrm{mm}^{2}\right)$ in each section are reported. (D) VSM cell marker $\alpha$-smooth muscle actin $(\alpha-$ SMA) is depicted $(n=10$ per group). Dashed line outlines the lesion area. Mean percent $\alpha$-SMA area relative to total lesion area is reported. Enlarged images (fourfold; upper right) represent the $\alpha$-SMA-positive area in the intimal region. (E) Masson's trichrome stain for collagen (blue, collagen; red, cytoplasm and muscle fibers) (control, $n=13$; $\mathrm{PAO}, n=9$ ). Mean percent collagen area relative to total lesion area is reported. Data are means \pm SEM. ${ }^{*} P<0.05$. ns, not significant. Control versus PAO: Mann-Whitney $U$ test was used for statistical analysis. Scale bars, $150 \mu \mathrm{m}$.

lesions. Quantitative analysis of ER stress by immunofluorescence staining showed a significant $(P<0.01)$ reduction in p-eIF $2 \alpha$ and ATF3 ( -42.5 and $-42.6 \%$, respectively) in the macrophage-rich regions of lesions after chronic PAO treatment when compared to the control group (Fig. 7, A and B, and fig. S6). Moreover, PAO supplementation reduced XBP1 splicing in the hearts from these mice (fig. S5D). These results are consistent with our earlier findings in macrophages in vitro and demonstrate the efficacy of oral PAO treatment in alleviating macrophage ER stress in atherosclerotic lesions in vivo.

We next asked whether PAO could inhibit lipid-induced inflammasome activation and secretion of IL- $1 \beta$ and IL-18 in vivo. Previous studies showed that the hyperlipidemic diet in $A p o E^{-/-}$ mice causes a marked induction of the inflammasome in the lesions and systemically, as evidenced by increased secretion of IL- $1 \beta$ and IL-18 $(43,74)$. We found that the intensity of immunofluorescence staining for IL-1 $\beta$ in atherosclerotic lesions was significantly $(P<0.05)$ reduced by PAO (-32.8\%), demonstrating effective suppression of the inflammasome by oral PAO treatment (Fig. 7C and fig. S6). Analysis of serum from mice supplemented for 4 weeks with PAO (threefold higher dosage than found in Western diet) showed a significant $(P<0.05)$ reduction in systemic IL-1 $\beta$ and IL-18 production ( -32.2 and $-26.6 \%$, respectively) by
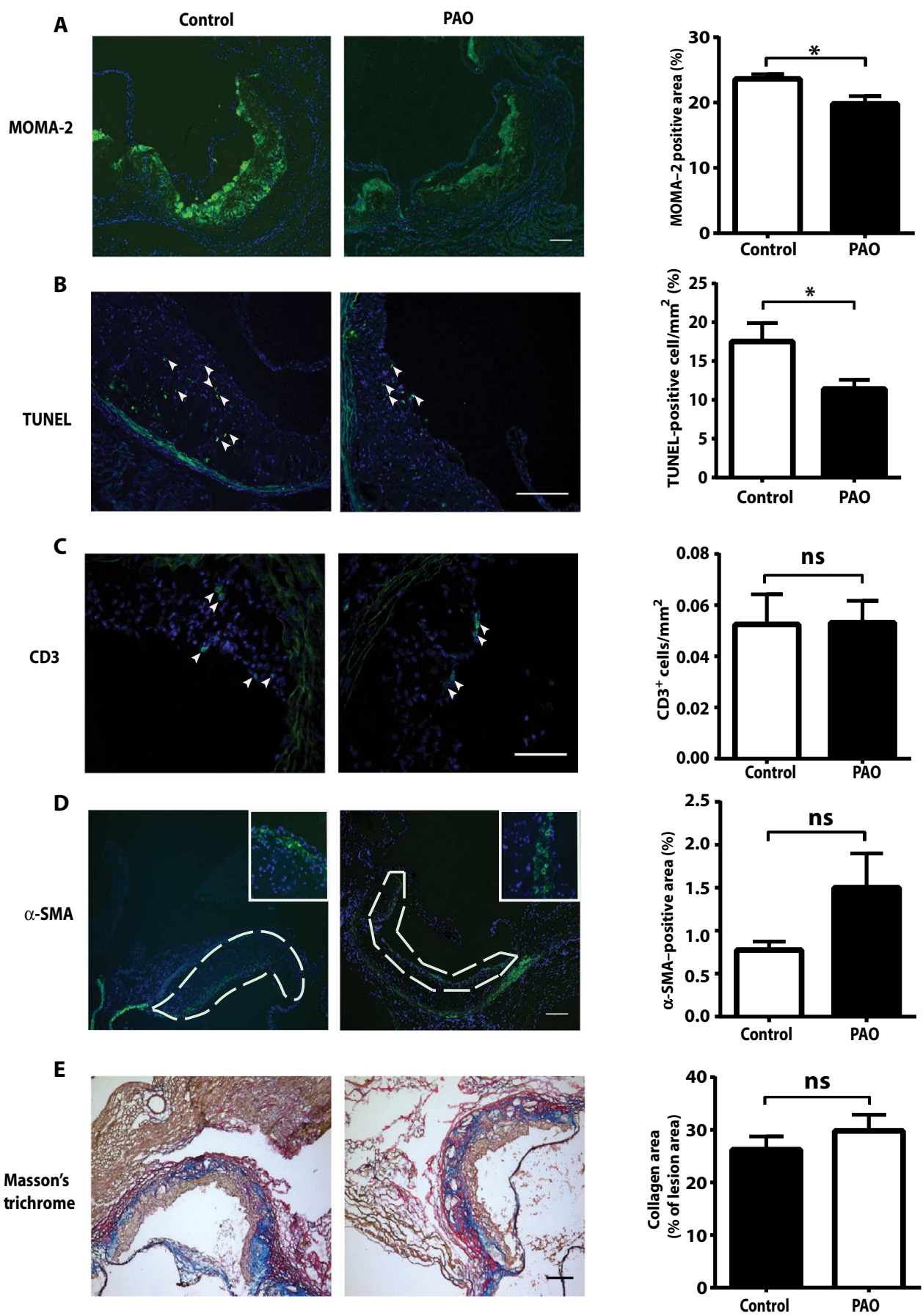

PAO treatment when compared to control mice (Fig. 7, D and E). Collectively, these findings demonstrate that chronic PAO supplementation can effectively block hyperlipidemia-induced macrophage ER stress and inflammasome activity in atherosclerotic plaques in vivo.

\section{DISCUSSION}

The contribution of DNL to obesity and its complications is complex in part because lipogenesis in various organs has different consequences 
on systemic metabolism. For example, liver DNL is increased in obesity and frequently associates with fatty liver disease and insulin resistance $(75,76)$. Lipogenesis in the skeletal muscle can promote insulin resistance but is also required for optimal muscle strength (77). Brain DNL generates lipid signals that affect food intake and energy metabolism (78). DNL in white adipose tissue is beneficial for systemic metabolism by increasing insulin sensitivity and improving lipid metabolism $(7,79)$. Therefore, developing therapeutic strategies based on DNL is challenging. For instance, a global DNL inhibitor may have beneficial metabolic effects acting on the hepatocytes, but adverse effects may be associated with blocking DNL in adipocytes, pancreatic $\beta$ cells, and macrophages (76). It is now clear that DNL can generate numerous lipid signals (1-palmitoyl-2-oleoylsn-glycerol-3-phosphocoline, alkyl ether lipids, and PAO) that can act through distinct receptors and mechanisms to exert potent metabolic effects (75). Alternatively, nutritional supplementation with a specific DNL product may effectively generate the beneficial metabolic effects of DNL in diseases afflicting target cells in metabolically active organs $(6,7,9-11,21,33,34,36,80)$. Despite a great interest in DNL-based therapeutic development for chronic metabolic diseases, DNL-generated lipid species' impact on the vasculature and atherosclerosis is not known. Circumstantial findings from several genetic or chemical models of DNL activation in mice suggest that DNL or its products could be protective against atherosclerosis $(33,34,36,75,79)$. Our study provides a direct examination of PAO's impact on atherogenesis. Our findings demonstrate that PAO supplementation (at threefold higher dosage than found in the composition of Western diet) reduces atherosclerosis in $\mathrm{ApoE}^{-/-}$mice. Chronic PAO treatment effectively mitigated macrophage ER stress in lesions and blocked inflammasome activation in lesions and systemically, similar to our initial observation in cultured primary mouse and human macrophages. In addition, a recent PAO supplementation trial in humans resulted in a marked reduction in high-sensitivity C-reactive protein and improved serum lipids, suggesting that PAO supplementation could also be atheroprotective in humans (36). Collectively, the outcome of these studies implies that nutritional supplementation with
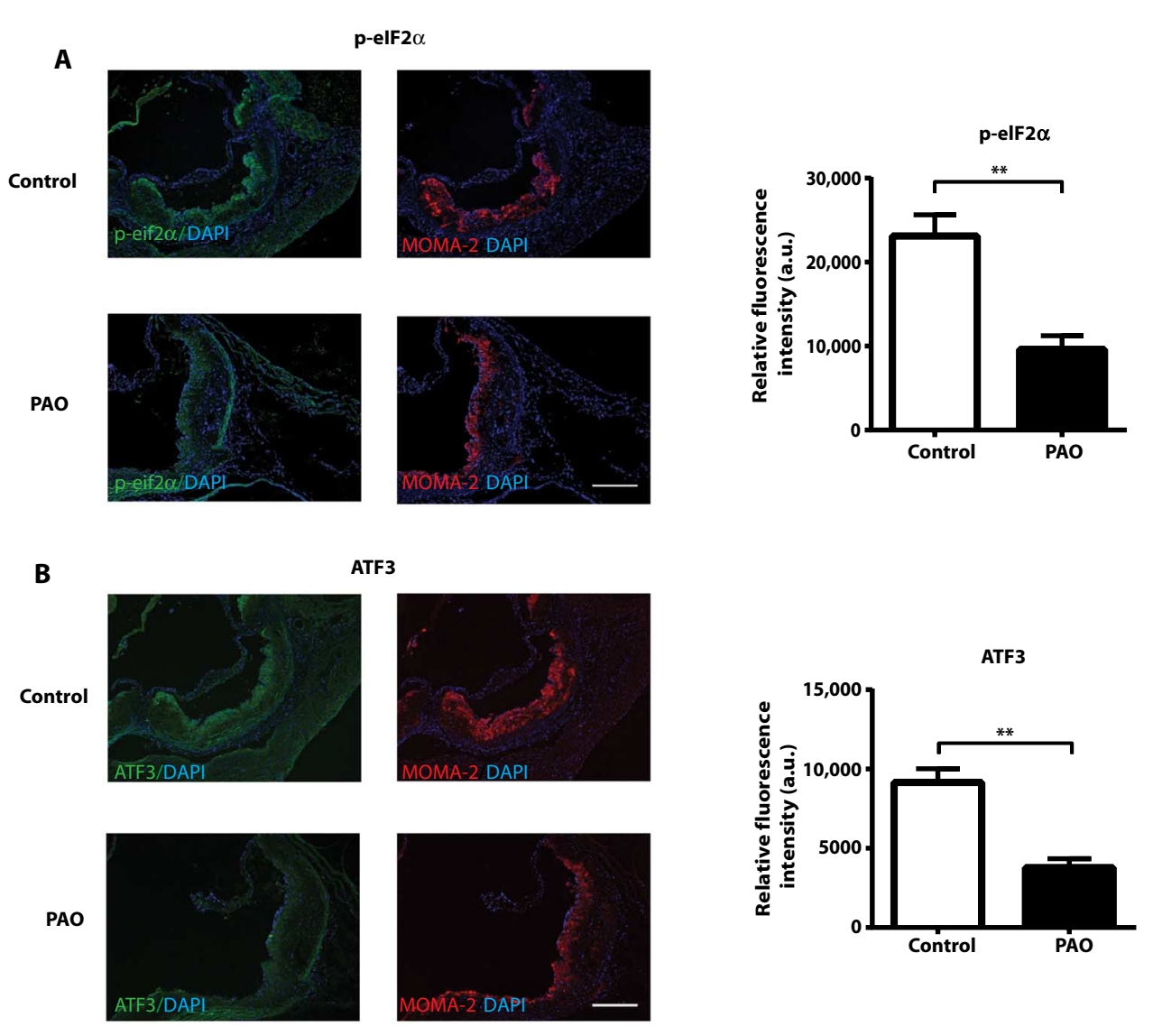

C
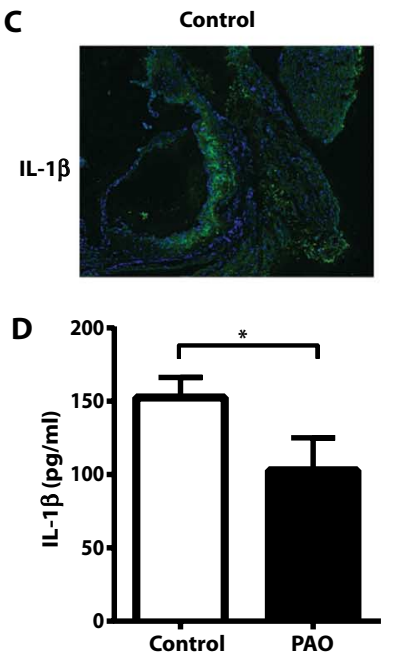

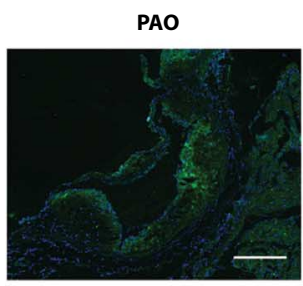

E

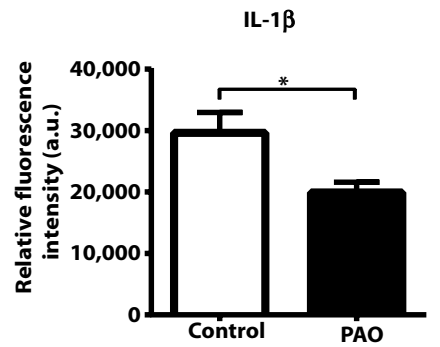

Fig. 7. PAO mitigates ER stress in lesional macrophages and suppresses the inflammasome in lesions. Aortic root sections from PAO or vehicle-treated mice were analyzed using the following specific antibodies for immunofluorescence staining: (A) p-elF2 $\alpha$ (green) and MOMA-2 (red) (control, $n=8 ; \mathrm{PAO}, n=$ 5), (B) ATF3 (green) and MOMA-2 (red) (control, $n=8$; PAO, $n=4$ ), and (C) IL-1 $\beta$ (control, $n=10 ;$ PAO, $n=8$ ). Fluorescence intensity of ER stress markers in macrophage-enriched (MOMA-2-positive) areas was quantified using ImageJ. IL-1 $\beta$ (control, $n=8 ;$ PAO, $n=4$ ) (D) and IL-18 ( $n=7$ per group) (E) were measured in plasma of $A p o E^{-1}$ mice treated with or without PAO after 4 weeks. All data are means $\pm \mathrm{SEM}$. ${ }^{*} P<0.05$, ${ }^{* *} P<$ 0.01. Control versus PAO: Mann-Whitney $U$ test was used for statistical analysis. Scale bars, $150 \mu \mathrm{m}$. a.u., arbitrary units. 
PAO may be a promising preventive approach in atherosclerosis through effective mitigation of ER stress and inflammasome activity. This does, however, require direct validation in future human trials.

The mechanism of action of DNL-produced lipids in vivo during atherogenesis is not known. Here, we sought to gain insight into PAO's mechanism of action in vivo by studying its effects on macrophages involved in atherogenesis. Macrophages play a prominent role in the progression of atherosclerosis to vulnerable plaques $(38,62)$. Previous studies established that cholesterol crystals and SFAs specifically induce NLRP3 inflammasome and IL-1 $\beta$ secretion from macrophages, and genetic or pharmacological inhibition of the inflammasome alleviates atherosclerosis $(43,74,81)$. Here, we found that treatment of primary mouse or human macrophages with PAO effectively blocked inflammasome activation by SFAs, but not by other NLRP3 inducers like ATP and alum. Additionally, PAO did not prevent activation of any other inflammasome complexes (AIM2, NLRC4, or NLRP1) by their specific inducers. These results underscore PAO's specificity as an inhibitor to lipid-induced NLRP3 inflammasome activation. Consistently, in vivo PAO treatment also lowered hyperlipidemia-induced IL-1 $\beta$ and IL-18 concentrations in serum and IL- $1 \beta$ expression in plaques. Therapies involving small-molecule inhibitors and dietary regimes aimed at blocking inflammasome activation and IL- $1 \beta$ secretion in a variety of diseases are highly sought after $(52,69,82)$.

The activation signal for the inflammasome can be generated by distress in $\operatorname{ER}(44,47,50,83)$. There are several ways in which ER can induce inflammasome activity, including release of $\mathrm{Ca}^{2+}$ or ROS and mitochondrial damage $(47,49)$. Moreover, damaged mitochondria generate ROS, which can further potentiate inflammasome activity (49). In an earlier study, we showed that PAO is a potent inhibitor of lipidinduced but not proteotoxic ER stress (11). Studies have also shown ER stress being causally associated with atherogenesis, diabetes, and obesity (84). Here, we confirmed that PAO inhibits hyperlipidemia-induced ER stress in vivo and in the macrophages infiltrating atherosclerotic plaques, and subsequent inflammasome activation. Collectively, the outcome of these experiments underscores PAO's ability to strongly antagonize lipid-induced ER stress upstream of inflammasome activation.

How does PAO promote metabolic resilience to hyperlipidemia? Dietary PAO intake is quite low because PAO is not ubiquitiously found in food sources (25). However, PAO is the fifth most abundant FA in humans, suggesting that endogenous PAO synthesis, particularly in obesity, is a major contributor to PAO abundance in humans (85). One plausible explanation for the increased PAO production in obesity is that it reflects a necessary metabolic adaptation to meet the high demand for MUFA in rerouting the metabolism of SFA and cholesterol toward storage or excretion and to support membrane function by active remodeling (86-89). Here, we showed that PAO effectively replaces other FAs, resulting in increased MUFA/SFA ratio in macrophages, plasma, and aorta, but without expanding any of the lipid classes. The marked incorporation of PAO into multiple PL species and, in particular, cardiolipin implies dynamic membrane remodeling of organelles through desaturation by PAO. Further lipidomic analysis of isolated ER fractions from macrophages and livers confirmed that PAO effectively incorporates into most lipid compartments, especially PLs and cholesterol, in this organelle and increases the MUFA/SFA ratio. Extensive ER membrane remodeling can thus be achieved through PAO-induced desaturation and may have wider implications, such as altered membrane biophysical properties and UPR signaling emanating from these membranes. Proximal UPR regulators form high-order oligomers on ER membranes upon ER stress induction by chemicals (61). Here, we show that PA evokes IRE1 oligomerization on ER membranes and splicing of XBP1 mRNA, which can be blocked by PAO cotreatment. The outcome of these experiments strongly supports the idea that dynamic remodeling of membranes by desaturation with PAO underlies ER resistance to stress induced by PA. One limitation of our study is that we do not know the extent of remodeling in other intracellular organelles' membranes because we only analyzed the ER via lipidomics. However, our study provides proof of principle that organelle membrane remodeling by nutritional supplementation with a bioactive lipid could be an effective strategy for the treatment of atherosclerosis.

This study and others support the conclusion that PAO supplementation can mitigate more than one component of metabolic syndrome, including insulin resistance and atherosclerosis $(7,28,33,36,90)$. PAO is a potent inhibitor of lipid-induced ER stress and inflammasome activation in mouse and human macrophages and atherosclerotic plaques in vivo. These beneficial changes are associated with dynamic lipidomic remodeling of ER membranes in mice in vivo. Our findings show that it is possible to modify organelle stress responses and suppress inflammasome activity through nutritional supplementation with PAO. PAO supplementation reduces atherosclerosis in mice, and these results warrant future investigation to assess PAO's therapeutic potential in human disease.

\section{SUPPLEMENTARY MATERIALS}

www.sciencetranslationalmedicine.org/cgi/content/full/8/358/358ra126/DC1 Materials and Methods

Fig. S1. Lipid-induced proinflammatory cytokine expression and ER stress prevented by PAO. Fig. S2. Increased desaturation of plasma lipids in PAO-supplemented $\mathrm{ApoE}^{-/}$mice.

Fig. S3. Lipid class composition analysis of plasma from $A p o E^{-1}$ mice.

Fig. S4. Analysis of liver or macrophage ER membrane lipid composition and IRE oligomerization on ER.

Fig. S5. Lipoprotein profile and ER stress status of PAO-supplemented $A p o E^{-1-}$ mice.

Fig. S6. Representative images for negative controls in immunofluorescence staining.

Table S1. Physical and biochemical characteristics of $\mathrm{ApoE}^{-/-}$mice in PAO-treated and control groups.

Table S2. Raw data and statistical analyses (provided as an Excel file).

References (96-99)

\section{REFERENCES AND NOTES}

1. A. Aarsland, D. Chinkes, R. R. Wolfe, Contributions of de novo synthesis of fatty acids to total VLDL-triglyceride secretion during prolonged hyperglycemia/hyperinsulinemia in normal man. J. Clin. Invest. 98, 2008-2017 (1996).

2. E. J. Parks, M. K. Hellerstein, Carbohydrate-induced hypertriacylglycerolemia: Historical perspective and review of biological mechanisms. Am. J. Clin. Nutr. 71, 412-433 (2000).

3. L. Abu-Elheiga, M. M. Matzuk, P. Kordari, W. Oh, T. Shaikenov, Z. Gu, S. J. Wakil, Mutant mice lacking acetyl-CoA carboxylase 1 are embryonically lethal. Proc. Natl. Acad. Sci. U.S.A. 102, 12011-12016 (2005).

4. S. S. Chirala, H. Chang, M. Matzuk, L. Abu-Elheiga, J. Mao, K. Mahon, M. Finegold, S. J. Wakil, Fatty acid synthesis is essential in embryonic development: Fatty acid synthase null mutants and most of the heterozygotes die in utero. Proc. Natl. Acad. Sci. U.S.A. 100, 6358-6363 (2003).

5. M. V. Chakravarthy, I. J. Lodhi, L. Yin, R. R. Malapaka, H. E. Xu, J. Turk, C. F. Semenkovich, Identification of a physiologically relevant endogenous ligand for PPAR $\alpha$ in liver. Cell 138, 476-488 (2009).

6. S. Liu, J. D. Brown, K. J. Stanya, E. Homan, M. Leidl, K. Inouye, P. Bhargava, M. R. Gangl, L. Dai, B. Hatano, G. S. Hotamisligil, A. Saghatelian, J. Plutzky, C.-H. Lee, A diurnal serum lipid integrates hepatic lipogenesis and peripheral fatty acid use. Nature 502, 550-554 (2013).

7. H. Cao, K. Gerhold, J. R. Mayers, M. M. Wiest, S. M. Watkins, G. S. Hotamisligil, Identification of a lipokine, a lipid hormone linking adipose tissue to systemic metabolism. Cell 134, 933-944 (2008). 
8. I. J. Lodhi, L. Yin, A. P. L. Jensen-Urstad, K. Funai, T. Coleman, J. H. Baird, M. K. El Ramahi, B. Razani, H. Song, F. Fu-Hsu, J. Turk, C. F. Semenkovich, Inhibiting adipose tissue lipogenesis reprograms thermogenesis and PPAR $\gamma$ activation to decrease diet-induced obesity. Cell Metab. 16, 189-201 (2012).

9. M. M. Yore, I. Syed, P. M. Moraes-Vieira, T. Zhang, M. A. Herman, E. A. Homan, R. T. Patel, J. Lee, S. Chen, O. D. Peroni, A. S. Dhaneshwar, A. Hammarstedt, U. Smith, T. E. McGraw, A. Saghatelian, B. B. Kahn, Discovery of a class of endogenous mammalian lipids with anti-diabetic and anti-inflammatory effects. Cell 159, 318-332 (2014).

10. C. A. Riquelme, J. A. Magida, B. C. Harrison, C. E. Wall, T. G. Marr, S. M. Secor, L. A. Leinwand, Fatty acids identified in the Burmese python promote beneficial cardiac growth. Science 334, 528-531 (2011)

11. E. Erbay, V. R. Babaev, J. R. Mayers, L. Makowski, K. N. Charles, M. E. Snitow, S. Fazio, M. M. Wiest, S. M. Watkins, M. F. Linton, G. S. Hotamisligil, Reducing endoplasmic reticulum stress through a macrophage lipid chaperone alleviates atherosclerosis. Nat. Med. 15, 1383-1391 (2009).

12. G. S. Hotamisligil, R. S. Johnson, R. J. Distel, R. Ellis, V. E. Papaioannou, B. M. Spiegelman, Uncoupling of obesity from insulin resistance through a targeted mutation in $a P 2$, the adipocyte fatty acid binding protein. Science 274, 1377-1379 (1996).

13. L. Makowski, J. B. Boord, K. Maeda, V. R. Babaev, K. T. Uysal, M. A. Morgan, R. A. Parker, J. Suttles, S. Fazio, G. S. Hotamisligil, M. F. Linton, Lack of macrophage fatty-acid-binding protein aP2 protects mice deficient in apolipoprotein E against atherosclerosis. Nat. Med. 7, 699-705 (2001).

14. K. Maeda, H. Cao, K. Kono, C. Z. Gorgun, M. Furuhashi, K. T. Uysal, Q. Cao, G. Atsumi, H. Malone, B. Krishnan, Y. Minokoshi, B. B. Kahn, R. A. Parker, G. S. Hotamisligil, Adipocyte/macrophage fatty acid binding proteins control integrated metabolic responses in obesity and diabetes. Cell Metab. 1, 107-119 (2005).

15. H. Cao, K. Maeda, C. Z. Gorgun, H.-J. Kim, S.-Y. Park, G. I. Shulman, J. K. Kim, G. S. Hotamisligil, Regulation of metabolic responses by adipocyte/macrophage fatty acid-binding proteins in leptin-deficient mice. Diabetes 55, 1915-1922 (2006).

16. E. Shrago, J. A. Glennon, E. S. Gordon, Comparative aspects of lipogenesis in mammalian tissues. Metabolism 20, 54-62 (1971).

17. G. S. Hotamisligil, D. A. Bernlohr, Metabolic functions of FABPs-Mechanisms and therapeutic implications. Nat. Rev. Endocrinol. 11, 592-605 (2015).

18. L. Scheja, L. Makowski, K. T. Uysal, S. M. Wiesbrock, D. R. Shimshek, D. S. Meyers, M. Morgan R. A. Parker, G. S. Hotamisligil, Altered insulin secretion associated with reduced lipolytic efficiency in aP2-/- mice. Diabetes 48, 1987-1994 (1999).

19. Z.-H. Yang, H. Miyahara, A. Hatanaka, Chronic administration of palmitoleic acid reduces insulin resistance and hepatic lipid accumulation in KK- $\mathrm{A}^{\mathrm{y}}$ Mice with genetic type 2 diabetes. Lipids Health Dis. 10, 120 (2011).

20. S. K. Duckett, G. Volpi-Lagreca, M. Alende, N. M. Long, Palmitoleic acid reduces intramuscular lipid and restores insulin sensitivity in obese sheep. Diabetes Metab. Syndr. Obes. 7, 553-563 (2014).

21. Z.-H. Yang, J. Takeo, M. Katayama, Oral administration of omega-7 palmitoleic acid induces satiety and the release of appetite-related hormones in male rats. Appetite 65, 1-7 (2013)

22. X. Guo, H. Li, H. Xu, V. Halim, W. Zhang, H. Wang, K. T. Ong, S. L. Woo, R. L. Walzem, D. G. Mashek, H. Dong, F. Lu, L. Wei, Y. Huo, C. Wu, Palmitoleate induces hepatic steatosis but suppresses liver inflammatory response in mice. PLOS ONE 7, e39286 (2012).

23. P. Puri, M. M. Wiest, O. Cheung, F. Mirshahi, C. Sargeant, H.-K. Min, M. J. Contos, R. K. Sterling, M. Fuchs, H. Zhou, S. M. Watkins, A. J. Sanyal, The plasma lipidomic signature of nonalcoholic steatohepatitis. Hepatology 50, 1827-1838 (2009).

24. F. Paillard, D. Catheline, F. Le Duff, M. Bouriel, Y. Deugnier, M. Pouchard, J.-C. Daubert P. Legrand, Plasma palmitoleic acid, a product of stearoyl-coA desaturase activity, is an independent marker of triglyceridemia and abdominal adiposity. Nutr. Metab. Cardiovasc. Dis. 18, 436-440 (2008)

25. L. Hodson, F. Karpe, Is there something special about palmitoleate? Curr. Opin. Clin. Nutr. Metab. Care 16, 225-231 (2013)

26. S. Fu, S. M. Watkins, G. S. Hotamisligil, The role of endoplasmic reticulum in hepatic lipid homeostasis and stress signaling. Cell Metab. 15, 623-634 (2012).

27. J. E. Schaffer, Lipotoxicity: When tissues overeat. Curr. Opin. Lipidol. 14, 281-287 (2003).

28. N. Stefan, K. Kantartzis, N. Celebi, H. Staiger, J. Machann, F. Schick, A. Cegan, M. Elcnerova, E. Schleicher, A. Fritsche, H.-U. Häring, Circulating palmitoleate strongly and independently predicts insulin sensitivity in humans. Diabetes Care 33, 405-407 (2010).

29. L. Wang, A. R. Folsom, Z. J. Zheng, J. S. Pankow, J. H. Eckfeldt; ARIC Study Investigators, Plasma fatty acid composition and incidence of diabetes in middle-aged adults: The Atherosclerosis Risk in Communities (ARIC) Study. Am. J. Clin. Nutr. 78, 91-98 (2003).

30. B. Vessby, A. Aro, E. Skarfors, L. Berglund, I. Salminen, H. Lithell, The risk to develop NIDDM is related to the fatty acid composition of the serum cholesterol esters. Diabetes $\mathbf{4 3}, 1353-1357$ (1994).

31. K. E. Pinnick, M. J. Neville, B. A. Fielding, K. N. Frayn, F. Karpe, L. Hodson, Gluteofemoral adipose tissue plays a major role in production of the lipokine palmitoleate in humans. Diabetes 61, 1399-1403 (2012).
32. R. Roberts, L. Hodson, A. L. Dennis, M. J. Neville, S. M. Humphreys, K. E. Harnden, K. J. Micklem, K. N. Frayn, Markers of de novo lipogenesis in adipose tissue: Associations with small adipocytes and insulin sensitivity in humans. Diabetologia 52, 882-890 (2009).

33. C. O. Souza, A. A. Teixeira, E. A. Lima, H. A. Batatinha, L. M. Gomes, M. Carvalho-Silva, I. T. Mota, E. L. Streck, S. M. Hirabara, J. C. Rosa Neto, Palmitoleic acid (n-7) attenuates the immunometabolic disturbances caused by a high-fat diet independently of PPAR $\alpha$. Mediators Inflamm 2014, 582197 (2014)

34. K. L. Chan, N. J. Pillon, D. M. Sivaloganathan, S. R. Costford, Z. Liu, M. Théret, B. Chazaud, A. Klip, Palmitoleate reverses high fat-induced proinflammatory macrophage polarization via AMPactivated protein kinase (AMPK). J. Biol. Chem. 290, 16979-16988 (2015).

35. N. A. Talbot, C. P. Wheeler-Jones, M. E. Cleasby, Palmitoleic acid prevents palmitic acidinduced macrophage activation and consequent p38 MAPK-mediated skeletal muscle insulin resistance. Mol. Cell. Endocrinol. 393, 129-142 (2014).

36. A. M. Bernstein, M. F. Roizen, L. Martinez, Purified palmitoleic acid for the reduction of high-sensitivity C-reactive protein and serum lipids: A double-blinded, randomized, placebo controlled study. J. Clin. Lipidol. 8, 612-617 (2014).

37. C. Weber, A. Zernecke, P. Libby, The multifaceted contributions of leukocyte subsets to atherosclerosis: Lessons from mouse models. Nat. Rev. Immunol. 8, 802-815 (2008).

38. C. Weber, H. Noels, Atherosclerosis: Current pathogenesis and therapeutic options. Nat Med. 17, 1410-1422 (2011).

39. K. J. Moore, F. J. Sheedy, E. A. Fisher, Macrophages in atherosclerosis: A dynamic balance. Nat. Rev. Immunol. 13, 709-721 (2013).

40. J. Bernhagen, R. Krohn, H. Lue, J. L. Gregory, A. Zernecke, R. R. Koenen, M. Dewor, I. Georgiev A. Schober, L. Leng, T. Kooistra, G. Fingerle-Rowson, P. Ghezzi, R. Kleemann, S. R. McColl, R. Bucala, M. J. Hickey, C. Weber, MIF is a noncognate ligand of CXC chemokine receptors in inflammatory and atherogenic cell recruitment. Nat. Med. 13, 587-596 (2007).

41. R. R. Koenen, P. von Hundelshausen, I. V. Nesmelova, A. Zernecke, E. A. Liehn, A. Sarabi, B. K. Kramp, A. M. Piccinini, S. R. Paludan, M. A. Kowalska, A. J. Kungl, T. M. Hackeng, K. H. Mayo, C. Weber, Disrupting functional interactions between platelet chemokines inhibits atherosclerosis in hyperlipidemic mice. Nat. Med. 15, 97-103 (2009).

42. R. R. Koenen, C. Weber, Therapeutic targeting of chemokine interactions in atherosclerosis. Nat. Rev. Drug Discov. 9, 141-153 (2010).

43. P. Duewell, H. Kono, K. J. Rayner, C. M. Sirois, G. Vladimer, F. G. Bauernfeind, G. S. Abela, L. Franchi, G. Nunez, M. Schnurr, T. Espevik, E. Lien, K. A. Fitzgerald, K. L. Rock, K. J. Moore, S. D. Wright, V. Hornung, E. Latz, NLRP3 inflammasomes are required for atherogenesis and activated by cholesterol crystals. Nature 464, 1357-1361 (2010).

44. H. Wen, D. Gris, Y. Lei, S. Jha, L. Zhang, M. T.-H. Huang, W. J. Brickey, J. P.-Y. Ting, Fatty acidinduced NLRP3-PYCARD inflammasome activation interferes with insulin signaling. Nat Immunol. 12, 408-415 (2011).

45. K. Schroder, J. Tschopp, The inflammasomes. Cell 140, 821-832 (2010).

46. M. F. Gregor, G. S. Hotamisligil, Inflammatory mechanisms in obesity. Annu. Rev. Immunol. 29, 415-445 (2011).

47. T. Horng, Calcium signaling and mitochondrial destabilization in the triggering of the NLRP3 inflammasome. Trends Immunol. 35, 253-261 (2014).

48. Y. Wang, G. Z. Wang, P. S. Rabinovitch, I. Tabas, Macrophage mitochondrial oxidative stress promotes atherosclerosis and nuclear factor- $\mathrm{KB}$-mediated inflammation in macrophages. Circ. Res. 114, 421-433 (2014).

49. A. Abderrazak, T. Syrovets, D. Couchie, K. El Hadri, B. Friguet, T. Simmet, M. Rouis, NLRP3 inflammasome: From a danger signal sensor to a regulatory node of oxidative stress and inflammatory diseases. Redox Biol. 4, 296-307 (2015).

50. T. Strowig, J. Henao-Mejia, E. Elinav, R. Flavell, Inflammasomes in health and disease. Nature 481, 278-286 (2012)

51. L. L'Homme, N. Esser, L. Riva, A. Scheen, N. Paquot, J. Piette, S. Legrand-Poels, Unsaturated fatty acids prevent activation of NLRP3 inflammasome in human monocytes/macrophages. J. Lipid Res. 54, 2998-3008 (2013).

52. H. Guo, J. B. Callaway, J. P.-Y. Ting, Inflammasomes: Mechanism of action, role in disease, and therapeutics. Nat. Med. 21, 677-687 (2015).

53. E. Diakogiannaki, N. G. Morgan, Differential regulation of the ER stress response by long-chain fatty acids in the pancreatic $\beta$-cell. Biochem. Soc. Trans. 36, 959-962 (2008).

54. R. Sriburi, H. Bommiasamy, G. L. Buldak, G. R. Robbins, M. Frank, S. Jackowski, J. W. Brewer, Coordinate regulation of phospholipid biosynthesis and secretory pathway gene expression in XBP-1(S)-induced endoplasmic reticulum biogenesis. J. Biol. Chem. 282, 7024-7034 (2007).

55. I. Tabas, Cholesterol and phospholipid metabolism in macrophages. Biochim. Biophys. Acto 1529, 164-174 (2000)

56. R. Harmancey, C. R. Wilson, N. R. Wright, H. Taegtmeyer, Western diet changes cardiac acyl-CoA composition in obese rats: A potential role for hepatic lipogenesis. J. Lipid Res. 51, 1380-1393 (2010).

57. N. M. Borradaile, X. Han, J. D. Harp, S. E. Gale, D. S. Ory, J. E. Schaffer, Disruption of endoplasmic reticulum structure and integrity in lipotoxic cell death. J. Lipid Res. 47, 2726-2737 (2006). 
58. B. Feng, P. M. Yao, Y. Li, C. M. Devlin, D. Zhang, H. P. Harding, M. Sweeney, J. X. Rong, G. Kuriakose, E. A. Fisher, A. R. Marks, D. Ron, I. Tabas, The endoplasmic reticulum is the site of cholesterol-induced cytotoxicity in macrophages. Nat. Cell Biol. 5, 781-792 (2003).

59. A. V. Korennykh, P. F. Egea, A. A. Korostelev, J. Finer-Moore, C. Zhang, K. M. Shokat R. M. Stroud, P. Walter, The unfolded protein response signals through high-order assembly of Ire1. Nature 457, 687-693 (2009).

60. R. Volmer, K. van der Ploeg, D. Ron, Membrane lipid saturation activates endoplasmic reticulum unfolded protein response transducers through their transmembrane domains. Proc. Natl. Acad. Sci. U.S.A. 110, 4628-4633 (2013).

61. H. Li, A. V. Korennykh, S. L. Behrman, P. Walter, Mammalian endoplasmic reticulum stress sensor IRE1 signals by dynamic clustering. Proc. Natl. Acad. Sci. U.S.A. 107, 16113-16118 (2010).

62. T. Seimon, I. Tabas, Mechanisms and consequences of macrophage apoptosis in atherosclerosis. J. Lipid Res. 50, S382-S387 (2009).

63. S. Han, C.-P. Liang, T. DeVries-Seimon, M. Ranalletta, C. L. Welch, K. Collins-Fletcher, D. Accili, I. Tabas, A. R. Tall, Macrophage insulin receptor deficiency increases ER stress-induced apoptosis and necrotic core formation in advanced atherosclerotic lesions. Cell Metab. 3, 257-266 (2006).

64. A. J. Bowes, M. I. Khan, Y. Shi, L. Robertson, G. H. Werstuck, Valproate attenuates accelerated atherosclerosis in hyperglycemic apoE-deficient mice: Evidence in support of a role for endoplasmic reticulum stress and glycogen synthase kinase-3 in lesion development and hepatic steatosis. Am. J. Pathol. 174, 330-342 (2009).

65. M. Myoishi, H. Hao, T. Minamino, K. Watanabe, K. Nishihira, K. Hatakeyama, Y. Asada, K.-i. Okada, H. Ishibashi-Ueda, G. Gabbiani, M.-L. Bochaton-Piallat, N. Mochizuki, M. Kitakaze, Increased endoplasmic reticulum stress in atherosclerotic plaques associated with acute coronary syndrome. Circulation 116, 1226-1233 (2007).

66. A. X. Zhou, I. Tabas, The UPR in atherosclerosis. Semin. Immunopathol. 35, 321-332 (2013).

67. F. Engin, G. S. Hotamisligil, Restoring endoplasmic reticulum function by chemical chaperones: An emerging therapeutic approach for metabolic diseases. Diabetes Obes. Metab. 12 (suppl. 2), 108-115 (2010).

68. A. R. Tall, L. Yvan-Charvet, Cholesterol, inflammation and innate immunity. Nat. Rev. Immunol. 15, 104-116 (2015).

69. R. C. Coll, A. A. B. Robertson, J. J. Chae, S. C. Higgins, R. Muñoz-Planillo, M. C. Inserra, I. Vetter, L. S. Dungan, B. G. Monks, A. Stutz, D. E. Croker, M. S. Butler, M. Haneklaus, C. E. Sutton, G. Nuñez, E. Latz, D. L. Kastner, K. H. G. Mills, S. L. Masters, K. Schroder, M. A. Cooper, L. A. J. O'Neill, A small-molecule inhibitor of the NLRP3 inflammasome for the treatment of inflammatory diseases. Nat. Med. 21, 248-255 (2015).

70. O. M. Finucane, C. L. Lyons, A. M. Murphy, C. M. Reynolds, R. Klinger, N. P. Healy, A. A. Cooke, R. C. Coll, L. McAllan, K. N. Nilaweera, M. E. O'Reilly, A. C. Tierney, M. J. Morine, J. F. Alcala-Diaz, J. Lopez-Miranda, D. P. O'Connor, L. A. O'Neill, F. C. McGillicuddy, H. M. Roche, Monounsaturated fatty acid-enriched high-fat diets impede adipose NLRP3 inflammasomemediated IL-1 $\beta$ secretion and insulin resistance despite obesity. Diabetes $64,2116-2128$ (2015).

71. H. Kraskiewicz, U. FitzGerald, InterfERing with endoplasmic reticulum stress. Trends Pharmacol. Sci. 33, 53-63 (2011)

72. R. Ross, Atherosclerosis-An inflammatory disease. N. Engl. J. Med. 340, 115-126 (1999).

73. C. C. Hedrick, Lymphocytes in atherosclerosis. Arterioscler. Thromb. Vasc. Biol. 35, 253-257 (2015).

74. F. J. Sheedy, A. Grebe, K. J. Rayner, P. Kalantari, B. Ramkhelawon, S. B. Carpenter, C. E. Becker, H. N. Ediriweera, A. E. Mullick, D. T. Golenbock, L. M. Stuart, E. Latz, K. A. Fitzgerald, K. J. Moore, CD36 coordinates NLRP3 inflammasome activation by facilitating intracellular nucleation of soluble ligands into particulate ligands in sterile inflammation. Nat. Immunol. 14, 812-820 (2013).

75. S. Liu, R. K. Alexander, C.-H. Lee, Lipid metabolites as metabolic messengers in inter-organ communication. Trends Endocrinol. Metab. 25, 356-363 (2014).

76. G. Solinas, J. Borén, A. G. Dulloo, De novo lipogenesis in metabolic homeostasis: More friend than foe? Mol. Metab. 4, 367-377 (2015).

77. K. Funai, H. Song, L. Yin, I. J. Lodhi, X. Wei, J. Yoshino, T. Coleman, C. F. Semenkovich, Muscle lipogenesis balances insulin sensitivity and strength through calcium signaling. J. Clin. Invest. 123, 1229-1240 (2013).

78. M. Fantino, Role of lipids in the control of food intake. Curr. Opin. Clin. Nutr. Metab. Care 14, 138-144 (2011).

79. L. Eissing, T. Scherer, K. Tödter, U. Knippschild, J. W. Greve, W. A. Buurman, H. O. Pinnschmidt S. S. Rensen, A. M. Wolf, A. Bartelt, J. Heeren, C. Buettner, L. Scheja, De novo lipogenesis in human fat and liver is linked to ChREBP- $\beta$ and metabolic health. Nat. Commun. 4, 1528 (2013).

80. J. M. Lee, Y. K. Lee, J. L. Mamrosh, S. A. Busby, P. R. Griffin, M. C. Pathak, E. A. Ortlund, D. D. Moore, A nuclear-receptor-dependent phosphatidylcholine pathway with antidiabetic effects. Nature 474, 506-510 (2011)

81. A. Abderrazak, D. Couchie, D. F. D. Mahmood, R. Elhage, C. Vindis, M. Laffargue, V. Matéo, B. Büchele, M. R. Ayala, M. El Gaafary, T. Syrovets, M.-N. Slimane, B. Friguet, T. Fulop, T. Simmet,
K. El Hadri, M. Rouis, Anti-inflammatory and antiatherogenic effects of the NLRP3 inflammasome inhibitor arglabin in $\mathrm{ApoE}_{2}$.Ki mice fed a high-fat diet. Circulation 131, 1061-1070 (2015).

82. Y.-H. Youm, K. Y. Nguyen, R. W. Grant, E. L. Goldberg, M. Bodogai, D. Kim, D. D'Agostino, N. Planavsky, C. Lupfer, T. D. Kanneganti, S. Kang, T. L. Horvath, T. M. Fahmy, P. A. Crawford A. Biragyn, E. Alnemri, V. D. Dixit, The ketone metabolite $\beta$-hydroxybutyrate blocks NLRP3 inflammasome-mediated inflammatory disease. Nat. Med. 21, 263-269 (2015).

83. P. Menu, A. Mayor, R. Zhou, A. Tardivel, H. Ichijo, K. Mori, J. Tschopp, ER stress activates the NLRP3 inflammasome via an UPR-independent pathway. Cell Death Dis. 3, e261 (2012).

84. S. Hummasti, G. S. Hotamisligil, Endoplasmic reticulum stress and inflammation in obesity and diabetes. Circ. Res. 107, 579-591 (2010).

85. L. Hodson, C. M. Skeaff, B. A. Fielding, Fatty acid composition of adipose tissue and blood in humans and its use as a biomarker of dietary intake. Prog. Lipid Res. 47, 348-380 (2008).

86. M. T. Clandinin, S. Cheema, C. J. Field, M. L. Garg, J. Venkatraman, T. R. Clandinin, Dietary fat: Exogenous determination of membrane structure and cell function. FASEB J. 5, 2761-2769 (1991).

87. L. L. Listenberger, X. Han, S. E. Lewis, S. Cases, R. V. Farese Jr., D. S. Ory, J. E. Schaffer, Triglyceride accumulation protects against fatty acid-induced lipotoxicity. Proc. Natl. Acad. Sci. U.S.A. 100, 3077-3082 (2003).

88. A. G. Clamp, S. Ladha, D. C. Clark, R. F. Grimble, E. K. Lund, The influence of dietary lipids on the composition and membrane fluidity of rat hepatocyte plasma membrane. Lipids 32 179-184 (1997)

89. I. Tabas, S. Marathe, G. A. Keesler, N. Beatini, Y. Shiratori, Evidence that the initial up-regulation of phosphatidylcholine biosynthesis in free cholesterol-loaded macrophages is an adaptive response that prevents cholesterol-induced cellular necrosis. Proposed role of an eventual failure of this response in foam cell necrosis in advanced atherosclerosis. J. Biol. Chem. 271, 22773-22781 (1996).

90. D. Mozaffarian, H. Cao, I. B. King, R. N. Lemaitre, X. Song, D. S. Siscovick, G. S. Hotamisligil, Circulating palmitoleic acid and risk of metabolic abnormalities and new-onset diabetes. Am. J. Clin. Nutr. 92, 1350-1358 (2010).

91. V. A. K. Rathinam, Z. Jiang, S. N. Waggoner, S. Sharma, L. E. Cole, L. Waggoner, S. K. Vanaja, B. G. Monks, S. Ganesan, E. Latz, V. Hornung, S. N. Vogel, E. Szomolanyi-Tsuda, K. A. Fitzgerald, The AIM2 inflammasome is essential for host defense against cytosolic bacteria and DNA viruses. Nat. Immunol. 11, 395-402 (2010).

92. E. Kuroda, K. J. Ishii, S. Uematsu, K. Ohata, C. Coban, S. Akira, K. Aritake, Y. Urade, Y. Morimoto, Silica crystals and aluminum salts regulate the production of prostaglandin in macrophages via NALP3 inflammasome-independent mechanisms. Immunity 34, 514-526 (2011).

93. Y. Qu, S. Misaghi, K. Newton, L. L. Gilmour, S. Louie, J. E. Cupp, G. R. Dubyak, D. Hackos, V. M. Dixit, Pannexin-1 is required for ATP release during apoptosis but not for inflammasome activation. J. Immunol. 186, 6553-6561 (2011).

94. M. G. Netea, C. A. Nold-Petry, M. F. Nold, L. A. B. Joosten, B. Opitz, J. H. M. van der Meer, F. L. van de Veerdonk, G. Ferwerda, B. Heinhuis, I. Devesa, C. J. Funk, R. J. Mason, B. J. Kullberg, A. Rubartelli, J. W. M. van der Meer, C. A. Dinarello, Differential requirement for the activation of the inflammasome for processing and release of $\mathrm{IL}-1 \beta$ in monocytes and macrophages. Blood 113, 2324-2335 (2009).

95. G. M. Keegan, J. D. Smart, M. J. Ingram, L.-M. Barnes, G. R. Burnett, G. D. Rees, Chitosan microparticles for the controlled delivery of fluoride. J. Dent. 40, 229-240 (2012).

96. T. Nakamura, M. Furuhashi, P. Li, H. Cao, G. Tuncman, N. Sonenberg, C. Z. Gorgun, G. S. Hotamisligil, Double-stranded RNA-dependent protein kinase links pathogen sensing with stress and metabolic homeostasis. Cell 140, 338-348 (2010).

97. A. G. Lerner, J.-P. Upton, P. V. K. Praveen, R. Ghosh, Y. Nakagawa, A. Igbaria, S. Shen, V. Nguyen, B. J. Backes, M. Heiman, N. Heintz, P. Greengard, S. Hui, Q. Tang, A. Trusina, S. A. Oakes, F. R. Papa, IRE1 $\alpha$ induces thioredoxin-interacting protein to activate the NLRP3 inflammasome and promote programmed cell death under irremediable ER stress. Cell Metab. 16, 250-264 (2012).

98. S. Fu, L. Yang, P. Li, O. Hofmann, L. Dicker, W. Hide, X. Lin, S. M. Watkins, A. R. Ivanov, G. S. Hotamisligil, Aberrant lipid metabolism disrupts calcium homeostasis causing liver endoplasmic reticulum stress in obesity. Nature 473, 528-531 (2011).

99. M. Furuhashi, G. Tuncman, C. Z. Görgün, L. Makowski, G. Atsumi, E. Vaillancourt, K. Kono, V. R. Babaev, S. Fazio, M. F. Linton, R. Sulsky, J. A. Robl, R. A. Parker, G. S. Hotamisligil, Treatment of diabetes and atherosclerosis by inhibiting fatty-acid-binding protein aP2. Nature 447, 959-965 (2007).

Acknowledgments: We express our appreciation to B. Gültekin and S. B. Widenmaier for assistance with the studies, K. Claiborn for critical reading and editing of the manuscript, C. Son for valuable advice on confocal imaging and analysis, P. Walter for GFP-IRE1 and KDEL plasmids, R. G. Roeder for the anti-TBP antibody, and Metabolon for lipidomic analysis. Funding: Marie Curie Reintegration Grant (Lipokines 276922). Author contributions: I.Ç. performed the 
in vivo mouse atherosclerosis study, en face aorta staining, cytokine ELISAs, cell culture experiments, and qRT-PCRs. B.K. performed immunofluorescence and collagen staining of lesions. S.K. and Ö.T. performed cell culture experiments related to inflammasomes, qRT-PCRs, and Western blots. O.A. performed immunofluorescence staining of lesions and helped with figure preparation. U.I.O. performed cell culture experiments and confocal microscopy imaging. A.D.Y. performed IRE1 oligomerization assays. Z.G.A. performed atherosclerosis experiments. Ş.D. performed cell culture experiments and qRT-PCR for inflammatory markers. I.Ç., B.K., and S.K. contributed to the writing of the manuscript. U.T.N. and S.M.W. analyzed the lipidomics data and critically read and edited the manuscript. G.S.H. analyzed the data and critically read and edited the manuscript. E.E. conceptualized the project, designed and directed the experiments, analyzed the data, and wrote the manuscript. Competing interests: G.S.H. received funding from UCB (Union Chimique Belge) and Servier for other projects, which was not used for the current study. S.M.W. and U.T.N. are employees of Metabolon, which carried out the lipidomic analysis.

Submitted 19 April 2016

Accepted 9 August 2016

Published 28 September 2016

10.1126/scitransImed.aaf9087

Citation: I. Çimen, B. Kocatürk, S. Koyuncu, Ö. Tufanlı, U. I. Onat, A. D. Yıldırım, O. Apaydın, Ş. Demirsoy, Z. G. Aykut, U. T. Nguyen, S. M. Watkins, G. S. Hotamışlıgil, E. Erbay, Prevention of atherosclerosis by bioactive palmitoleate through suppression of organelle stress and inflammasome activation. Sci. Transl. Med. 8, 358ra126 (2016). 


\section{Science Translational Medicine}

\section{Prevention of atherosclerosis by bioactive palmitoleate through suppression of organelle stress and inflammasome activation}

Ismail Çimen, Begüm Kocatürk, Seda Koyuncu, Özlem Tufanli, Umut I. Onat, Asli D. Yildirim, Onur Apaydin, Seyma Demirsoy, Zaliha G. Aykut, Uyen T. Nguyen, Steven M. Watkins, Gökhan S. Hotamisligil and Ebru Erbay

Sci Transl Med 8, 358ra126358ra126.

DOI: 10.1126/scitranslmed.aaf9087

\section{Artery-saving fat}

Although fatty foods often have a bad reputation when it comes to atherosclerosis, a study by Çimen et al. identifies a type of fat that is not only harmless but also protective. Palmitoleate is a lipid that can be produced directly by the human body and is also found in a variety of foods, but only in small amounts. Using human cells and mouse models, the authors demonstrate that palmitoleate reduces metabolic stress in a variety of tissues as well as in atherosclerotic plaques and thus decreases the severity of atherosclerosis in mouse models.

ARTICLE TOOLS

SUPPLEMENTARY MATERIALS

RELATED

CONTENT

REFERENCES

PERMISSIONS http://stm.sciencemag.org/content/8/358/358ra126

http://stm.sciencemag.org/content/suppl/2016/09/26/8.358.358ra126.DC1

http://stm.sciencemag.org/content/scitransmed/8/333/333ra50.full http://stm.sciencemag.org/content/scitransmed/7/275/275ra20.full http://stm.sciencemag.org/content/scitransmed/6/239/239sr1.full http://stm.sciencemag.org/content/scitransmed/5/166/166ra1. full http://stm.sciencemag.org/content/scitransmed/9/398/eaal2658.full http://stm.sciencemag.org/content/scitransmed/9/407/eaad4000.full

This article cites 99 articles, 31 of which you can access for free http://stm.sciencemag.org/content/8/358/358ra126\#BIBL

http://www.sciencemag.org/help/reprints-and-permissions

Use of this article is subject to the Terms of Service

Science Translational Medicine (ISSN 1946-6242) is published by the American Association for the Advancement of Science, 1200 New York Avenue NW, Washington, DC 20005. 2017 () The Authors, some rights reserved; exclusive licensee American Association for the Advancement of Science. No claim to original U.S. Government Works. The title Science Translational Medicine is a registered trademark of AAAS. 